JOURNAL OF THE

AMERICAN MATHEMATICAL SOCIETY

Volume 18, Number 3, Pages 735-761

S 0894-0347(05)00485-6

Article electronically published on April 8, 2005

\title{
A COMBINATORIAL FORMULA FOR MACDONALD POLYNOMIALS
}

\author{
J. HAGLUND, M. HAIMAN, AND N. LOEHR
}

\section{INTRODUCTION}

The Macdonald polynomials $\tilde{H}_{\mu}(x ; q, t)$ have been the subject of much attention in combinatorics since Macdonald [25] defined them and conjectured that their expansion in terms of Schur polynomials should have positive coefficients. Macdonald's conjecture was proven in [11] by geometric and representation-theoretic means, but these results do not provide any purely combinatorial interpretation for $\tilde{H}_{\mu}(x ; q, t)$. Such an interpretation, which had been sought for many years, was recently conjectured by Haglund [8. The goal of this paper is to prove the validity of Haglund's conjectured formula.

A number of consequences flow from the new formula and its proof. We shall summarize a few of them here. Some follow instantly, and the rest will be discussed in more detail in later sections of the paper.

(i) The Macdonald polynomials $\tilde{H}_{\mu}(x ; q, t)$ are characterized by certain axioms (see below). Their existence is not obvious from the axioms. To prove our combinatorial formula, we will show directly that it satisfies the axioms. Therefore we get a new proof of the existence theorem.

(ii) By definition, the coefficients of the Macdonald polynomials $\tilde{H}_{\mu}(x ; q, t)$ belong to the field of rational functions $\mathbb{Q}(q, t)$. In fact, the coefficients belong to $\mathbb{Z}[q, t]$. This integrality property was not proven until six or seven years after Macdonald formulated his conjecture, although many different proofs have since been found $[5,6,7,15,16,19,28$. Since our combinatorial formula is manifestly a polynomial, we get a new proof of integrality.

(iii) The celebrated formula of Lascoux and Schützenberger [21 for the expansion of Hall-Littlewood polynomials in terms of Schur functions is a corollary to our formula. In our setting, the charge, an intricate combinatorial statistic appearing in the Lascoux-Schützenberger formula, emerges naturally from simpler concepts.

(iv) The combinatorial formula of Knop and Sahi 17 for the Jack polynomials is a corollary to our formula. In fact, our formula yields a lift of the Knop-Sahi formula from Jack polynomials $J_{\mu}^{(\alpha)}(x)$ to integral form Macdonald polynomials $J_{\mu}(x ; q, t)$. (The Jack polynomial is the specialization $J_{\mu}^{(\alpha)}(x)=\lim _{t \rightarrow 1} J_{\mu}\left(x ; t^{\alpha}, t\right) /(1-t)^{|\mu|}$.)

Received by the editors October 18, 2004.

2000 Mathematics Subject Classification. Primary 05E10; Secondary 05A30.

The first author's work was supported by NSA grant MSPF-02G-193.

The second author's work was supported by NSF grant DMS-0301072.

The third author's work was supported by an NSF Postdoctoral Research Fellowship. 
(v) Our formula can be interpreted as expressing $\tilde{H}_{\mu}(x ; q, t)$ in terms of LLT polynomials, the symmetric functions involving one parameter $q$ introduced by Lascoux, Leclerc and Thibon [20]. The contact between Macdonald and LLT polynomials first seen in our earlier work with Remmel and Ulyanov [9] is thereby made stronger. We remark that the conjecture formulated in [9] led the first author to the formula established in this paper.

(vi) When the diagram of the partition $\mu$ has two columns, we obtain a new combinatorial formula for the coefficients $\tilde{K}_{\lambda \mu}(q, t)$ in the expansion of $\tilde{H}_{\mu}(x ; q, t)$ in terms of Schur polynomials $s_{\lambda}(x)$. It appears to be different from other combinatorial formulas that are known in the two-column case [4, 18, 32.

(vii) We hope that our formula may eventually lead to a combinatorial formula for $\tilde{K}_{\lambda \mu}(q, t)$ for general $\mu$, and so to a combinatorial proof of the positivity theorem from [1] that $\tilde{K}_{\lambda \mu}(q, t)$ is a polynomial in $q$ and $t$ with non-negative coefficients. As things stand, our formula does not yet solve this problem, because it expresses $\tilde{H}_{\mu}(x ; q, t)$ in terms of monomials, rather than Schur polynomials. Our formula does, however, reduce the problem to a special case of the conjecture in [20] that LLT polynomials have positive expansions in terms of Schur polynomials. That conjecture is known to hold for LLT polynomials indexed by tuples of partition diagrams [9, 24]. The case required for Macdonald positivity is that of a tuple of ribbon skew diagrams (see $\$ 3$ ).

We now recall the definition of Macdonald polynomials and indicate the plan of the paper. We mostly follow the notation in Macdonald's book [26] concerning partitions, symmetric functions, and so forth. We work in the algebra $\Lambda=\Lambda_{\mathbb{Q}(q, t)}(x)$ of formal symmetric functions in infinitely many variables $x=x_{1}, x_{2}, \ldots$, with coefficients in $\mathbb{Q}(q, t)$. Several bases of $\Lambda$ are the power-sums $p_{\mu}(x)$, the monomial symmetric functions $m_{\mu}(x)$, the elementary symmetric functions $e_{\mu}(x)$, the complete homogeneous symmetric functions $h_{\mu}(x)$, and the Schur functions $s_{\mu}(x)$. The basis element indexed by a partition $\mu$ in each case is homogeneous of degree $n=|\mu|$. We write $\langle-,-\rangle$ for the Hall scalar product

$$
\left\langle m_{\mu}, h_{\nu}\right\rangle=\delta_{\mu \nu}=\left\langle s_{\mu}, s_{\nu}\right\rangle
$$

and $\omega$ for the involutory automorphism of $\Lambda$

$$
\omega\left(e_{\mu}\right)=h_{\mu} ; \quad \omega\left(h_{\mu}\right)=e_{\mu} ; \quad \omega\left(p_{\mu}\right)=(-1)^{|\mu|-l(\mu)} p_{\mu} ; \quad \omega\left(s_{\mu}\right)=s_{\mu^{\prime}} .
$$

Here and throughout, $\mu^{\prime}$ denotes the transpose of $\mu$. The partitions of a given $n$ are partially ordered by

$$
\mu \leq \nu \quad \text { if } \quad \mu_{1}+\cdots+\mu_{k} \leq \nu_{1}+\cdots+\nu_{k} \quad \text { for all } k .
$$

If $A$ is a polynomial or formal series, $p_{k}[A]$ denotes the result of substituting $a^{k}$ for each indeterminate $a$ appearing in $A$ (including $q$ and $t$ ). For arbitrary $f \in \Lambda$, the plethystic substitution $f[A]$ is the result of expressing $f$ as a polynomial in the power-sums $p_{k}$ and substituting $p_{k}[A]$ for $p_{k}$ in $f$. By convention, we set $X=x_{1}+x_{2}+\cdots, Y=y_{1}+y_{2}+\cdots$. Then $f[X]=f(x), f[X+Y]=f(x, y)$, $f[-X]=(-1)^{d} \omega f(x)$ if $f$ is homogeneous of degree $d$, and $f[X(1-q)]$ is the image of $f$ under the algebra homomorphism mapping $p_{k}(x)$ to $\left(1-q^{k}\right) p_{k}(x)$. See, e.g., [10, §2] for a fuller account.

The Macdonald polynomials $\tilde{H}_{\mu}[Z ; q, t]$ are the basis of $\Lambda$ defined and characterized by the following triangularity and normalization axioms (see [10, Prop. 2.6] 
or [12, §6.1] for their equivalence with Macdonald's triangularity and orthogonality axioms).

$$
\begin{array}{ll}
\text { (T1) } & \tilde{H}_{\mu}[X(1-q) ; q, t]=\sum_{\lambda \geq \mu} a_{\lambda \mu}(q, t) s_{\lambda}, \\
\text { (T2) } & \tilde{H}_{\mu}[X(1-t) ; q, t]=\sum_{\lambda \geq \mu^{\prime}} b_{\lambda \mu}(q, t) s_{\lambda}, \\
\text { (N) } \quad\left\langle\tilde{H}_{\mu}, s_{(n)}\right\rangle=1,
\end{array}
$$

for suitable coefficients $a_{\lambda \mu}, b_{\lambda \mu} \in \mathbb{Q}(q, t)$. It is easy to see, as in [10, 12, that symmetric functions satisfying these axioms are unique if they exist. Their existence is equivalent to Macdonald's existence theorem in [25] and, as noted above, is also a corollary to the proof of our main theorem.

The main result of this paper (Theorem 2.2) is an identity $\tilde{H}_{\mu}(x ; q, t)=$ $C_{\mu}(x ; q, t)$, where the right-hand side is a purely combinatorial expression (Definition 2.1) given as the sum, over all $\mathbb{Z}_{+}$-valued functions $\sigma$ on the diagram of $\mu$, of a monomial $x^{\sigma}=\prod_{u} x_{\sigma(u)}$ multiplied by a suitable weight $q^{\operatorname{inv}(\sigma)} t^{\operatorname{maj}(\sigma)}$. The combinatorial statistics $\operatorname{inv}(\sigma)$ and $\operatorname{maj}(\sigma)$ are defined in $\$ 2$,

Theorem 2.2 is proven in \$5. The proof is a direct verification that the combinatorial expression $C_{\mu}(x ; q, t)$ satisfies the defining axioms (44) for $\tilde{H}_{\mu}(x ; q, t)$. The normalization axiom $(N)$ turns out to be trivial. Each of the two triangularity axioms (T1)-(T2) is proven with the aid of a suitable sign-reversing involution.

In order to interpret (T1)-(T2) for $C_{\mu}(x ; q, t)$ combinatorially, we must first show that $C_{\mu}(x ; q, t)$ is a symmetric function. This crucial result was announced by Haglund in [8]. We give its proof in $\$ 3$, using the theory of LLT polynomials. In the process, we obtain the LLT expansion of $C_{\mu}(x ; q, t)$, and hence of $\tilde{H}_{\mu}(x ; q, t)$, mentioned above under (v). This given, we can apply a standard technique of superization using quasisymmetric function expansions; this is explained in $\$$

Some of the consequences (i)-(vii) discussed above are further elaborated in $\S ₫ 6$ 9 especially those concerning the Lascoux-Schützenberger charge formula (\$77), the Knop-Sahi formula for Jack polynomials ( $(8)$, and the two-column case ( $(9)$ ).

Finally, in view of the important consequences of our main theorem on the one hand and the essential simplicity of its proof on the other, it was our desire to keep the reasoning in this paper self-contained, elementary and combinatorial. In fact, the only exception to these desiderata occurs in our reliance on the theory of LLT polynomials to establish the symmetry of $C_{\mu}(x ; q, t)$. Even this exception is removable, however, as we show in an appendix (\$10) where we provide a new, elementary proof of the symmetry theorem for LLT polynomials.

\section{THE FORMULA}

Let $\mu=\left(\mu_{1} \geq \mu_{2} \geq \cdots \geq \mu_{l}\right)$ be a partition of $n=\mu_{1}+\cdots+\mu_{l}$, and let

$$
\operatorname{dg}(\mu)=\left\{(i, j) \in \mathbb{Z}_{+} \times \mathbb{Z}_{+}: j \leq \mu_{i}\right\}
$$

be its Young (or Ferrers) diagram, whose elements are called cells. We draw diagrams in the first quadrant, French style, as

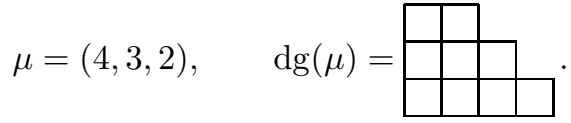


For simplicity, we henceforth write $\mu$ instead of $\operatorname{dg}(\mu)$ when it will not cause confusion. A filling is a function $\sigma: \mu \rightarrow \mathbb{Z}_{+}$, which we picture as assigning integer entries to the cells of $\mu$. We define

$$
x^{\sigma}=\prod_{u \in \mu} x_{\sigma(u)},
$$

a monomial of degree $n$ in the variables $x=x_{1}, x_{2}, \ldots$.

Haglund's formula gives the Macdonald polynomial as the sum of $q^{\operatorname{inv}(\sigma)} t^{\operatorname{maj}(\sigma)} x^{\sigma}$ over all fillings $\sigma: \mu \rightarrow \mathbb{Z}_{+}$, where $\operatorname{inv}(\sigma)$ and $\operatorname{maj}(\sigma)$ are simple combinatorial statistics, which we define next. A descent of $\sigma$ is a pair of entries $\sigma(u)>\sigma(v)$, where the cell $u$ is immediately above $v$, that is, $v=(i, j), u=(i+1, j)$. Define

$$
\operatorname{Des}(\sigma)=\{u \in \mu: \sigma(u)>\sigma(v) \text { is a descent }\} \text {. }
$$

The example below has two descents, as shown.

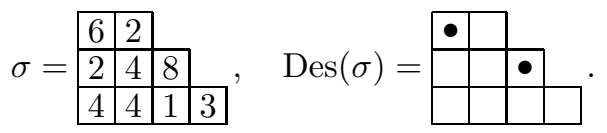

Two cells $u, v \in \mu$ are said to attack each other if either

(i) they are in the same row: $u=(i, j), v=(i, k)$; or

(ii) they are in consecutive rows, with the cell in the upper row strictly to the right of the one in the lower row: $u=(i+1, k), v=(i, j)$, where $j<k$.

The figure below shows the two types of pairs of attacking cells.

(i)

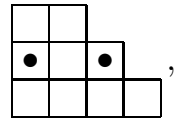

(ii)

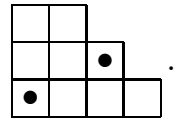

The reading order is the total ordering on the cells of $\mu$ given by reading them row by row, top to bottom, and left to right within each row. More formally, $(i, j)<\left(i^{\prime}, j^{\prime}\right)$ in the reading order if $(-i, j)$ is lexicographically less than $\left(-i^{\prime}, j^{\prime}\right)$. An inversion of $\sigma$ is a pair of entries $\sigma(u)>\sigma(v)$, where $u$ and $v$ attack each other and $u$ precedes $v$ in the reading order. Our example (9) has seven inversions: four in the bottom row and one in the top row, and two formed by the 8 in the second row attacking the two 4's in the bottom row. Define

$$
\operatorname{Inv}(\sigma)=\{\{u, v\}: \sigma(u)>\sigma(v) \text { is an inversion }\}
$$

Finally, the arm of a cell $u \in \mu$ is the number of cells strictly to the right of $u$ in the same row; its leg is the number of cells strictly above $u$ in the same column, as illustrated below.

$$
\begin{array}{|l|l|l|l}
\hline & l & & \\
\hline & l & & \\
\hline & \bullet & a & a
\end{array} \quad \operatorname{arm}(\bullet)=\operatorname{leg}(\bullet)=2 .
$$

Define

$$
\begin{aligned}
\operatorname{maj}(\sigma) & =\sum_{u \in \operatorname{Des}(\sigma)}(\operatorname{leg}(u)+1), \\
\operatorname{inv}(\sigma) & =|\operatorname{Inv}(\sigma)|-\sum_{u \in \operatorname{Des}(\sigma)} \operatorname{arm}(u) .
\end{aligned}
$$

Haglund's formula is as follows. 


\section{Definition 2.1.}

$$
C_{\mu}(x ; q, t)=\sum_{\sigma: \mu \rightarrow \mathbb{Z}_{+}} q^{\operatorname{inv}(\sigma)} t^{\operatorname{maj}(\sigma)} x^{\sigma} .
$$

Theorem 2.2. Formula (14) is equal to the Macdonald polynomial: $\tilde{H}_{\mu}(x ; q, t)=$ $C_{\mu}(x ; q, t)$.

In 8] it was observed that the statistic $\operatorname{inv}(\sigma)$ defined in (13) is always nonnegative. We recall the explanation, which we will need later. Three cells $u, v, w \in \mu$ are said to form a triple if they are situated as shown below,

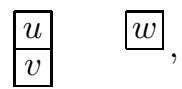

namely, $v$ is directly below $u$, and $w$ is in the same row as $u$, to its right. Define for $x, y \in \mathbb{Z}_{+}$

$$
I(x, y)= \begin{cases}1 & \text { if } x>y \\ 0 & \text { if } x \leq y\end{cases}
$$

Let $\sigma$ be a filling and let $x, y, z$ be the entries of $\sigma$ in the cells of a triple $(u, v, w)$ :

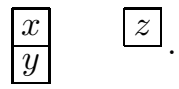

Then $I(x, y)=1$ if and only if $u \in \operatorname{Des}(\sigma)$, and $I(x, z)+I(z, y)$ is the contribution to $|\operatorname{Inv}(\sigma)|$ from the two attacking pairs $\{u, w\},\{v, w\}$. Note that every attacking pair either belongs to a unique triple or consists of two cells in the bottom row. The number of triples involving $u$ as their upper left cell is $\operatorname{arm}(u)$. Therefore

$$
\operatorname{inv}(\sigma)=|\operatorname{Inv}(\sigma)|-\sum_{u \in \operatorname{Des}(\sigma)} \operatorname{arm}(u)=J+\sum_{(u, v, w)} I(x, z)+I(z, y)-I(x, y)
$$

where $J$ is the number of inversions in the bottom row, the sum is over triples $(u, v, w)$ in $\mu$, and we denote $x=\sigma(u), y=\sigma(v), z=\sigma(w)$. The transitive law for < implies that $I(x, z)+I(z, y)-I(x, y) \in\{0,1\}$. Hence $\operatorname{inv}(\sigma)$ is non-negative, equal to $J$ plus the number of inversion triples in $\sigma$, defined as triples for which $I(x, z)+I(z, y)-I(x, y)=1$.

\section{LLT EXPANSION AND SYMMETRY}

Theorem 3.1. The polynomial $C_{\mu}(x ; q, t)$ is symmetric in the variables $x$.

We will prove Theorem 3.1 by expanding $C_{\mu}(x ; q, t)$ in terms of the remarkable symmetric functions defined by Lascoux, Leclerc and Thibon [20] and commonly known as LLT polynomials. We use here a variant definition of LLT polynomials introduced in 9 .

A skew diagram is a subset of $\mathbb{Z}_{+} \times \mathbb{Z}_{+}$of the form $\lambda \backslash \mu$, where $\lambda$ and $\mu$ are partition diagrams such that $\mu \subseteq \lambda$. The content of a cell $u=(i, j)$ in a skew diagram $\nu$ is the integer $c(u)=i-j$. So that $c(u)$ has a definite meaning, we do not follow the common practice of identifying skew diagrams that are translates of each other. As usual, a semistandard Young tableau of shape $\nu$ is a function $T: \nu \rightarrow \mathbb{Z}_{+}$which is weakly increasing on each row of $\nu$ and strictly increasing on 
each column. We denote the set of them by $\operatorname{SSYT}(\nu)$. Given $T \in \operatorname{SSYT}(\nu)$, define its monomial

$$
x^{T}=\prod_{u \in \nu} x_{T(u)} .
$$

Let

$$
\boldsymbol{\nu}=\left(\nu^{(1)}, \ldots, \nu^{(k)}\right)
$$

be a tuple of skew diagrams. We set $\operatorname{SSYT}(\boldsymbol{\nu})=\operatorname{SSYT}\left(\nu^{(1)}\right) \times \cdots \times \operatorname{SSYT}\left(\nu^{(k)}\right)$. Given $T=\left(T^{(1)}, \ldots, T^{(k)}\right) \in \operatorname{SSYT}(\boldsymbol{\nu})$, we set

$$
x^{T}=\prod_{i} x^{T^{(i)}} .
$$

Entries $T^{(i)}(u)>T^{(j)}(v)$ form an inversion if either

(i) $i<j$ and $c(u)=c(v)$, or

(ii) $i>j$ and $c(u)=c(v)+1$.

Denote by $\operatorname{inv}(T)$ the number of inversions in $T$.

Definition 3.2. The LLT polynomial indexed by $\boldsymbol{\nu}$ is

$$
G_{\boldsymbol{\nu}}(x ; q) \underset{\text { def }}{=} \sum_{T \in \operatorname{SSYT}(\boldsymbol{\nu})} q^{\operatorname{inv}(T)} x^{T} .
$$

Theorem $3.3([9,20])$. The polynomial $G_{\nu}(x ; q)$ is symmetric in the variables $x$.

Remark. The relationship between $G_{\nu}(x ; q)$ and the polynomial $\tilde{G}_{\lambda}^{(k)}(x ; q)$ defined in [20, eq. (27)] is as follows. In [20], $\lambda$ is a skew shape that can be tiled by $k$-ribbons. Our corresponding $\boldsymbol{\nu}$ is the $k$-quotient of $\lambda$. This given, $G_{\boldsymbol{\nu}}(x ; q)=q^{e} \tilde{G}_{\lambda}^{(k)}\left(x ; q^{-1}\right)$, where $e=\max _{T \in \operatorname{SSYT}(\boldsymbol{\nu})}(\operatorname{inv}(T))$. See [9, §5] for more details.

To relate formula (14) to the polynomials $G_{\boldsymbol{\nu}}(x ; q)$, we focus on the terms in (14) corresponding to fillings with a given descent set. For each subset $D \subseteq\{(i, j) \in \mu$ : $i>1\}$, define

$$
F_{\mu, D}(x ; q)=\sum_{\operatorname{Des}(\sigma)=D} q^{|\operatorname{Inv}(\sigma)|} x^{\sigma}
$$

Then, clearly,

$$
C_{\mu}(x ; q, t)=\sum_{D} q^{-\mathrm{a}(D)} t^{\operatorname{maj}(D)} F_{\mu, D}(x ; q),
$$

where $\mathrm{a}(D)=\sum_{u \in D} \operatorname{arm}(u)$ and $\operatorname{maj}(D)=\sum_{u \in D}(\operatorname{leg}(u)+1)$.

A ribbon is a connected skew shape containing no $2 \times 2$ block of cells, as shown:

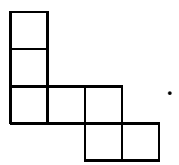

We only consider ribbons in fixed position such that the lower-right cell has content 1. Then the contents of all the cells are consecutive integers $1,2, \ldots, m$. Define the 
descent set of a ribbon $\nu$ to be the set of contents $c(u)$ of those cells $u=(i, j) \in \nu$ such that the cell $v=(i-1, j)$ directly below $u$ also belongs to $\nu$. In our example,

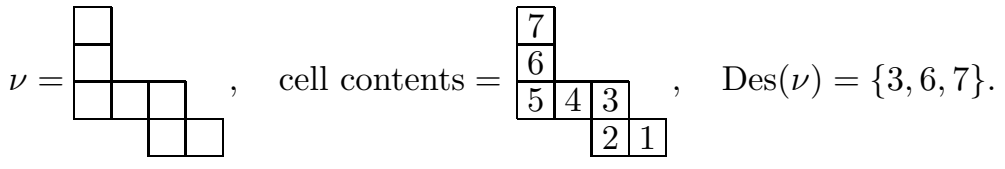

Clearly, we have a one-to-one correspondence between ribbons of size $m$ and descent sets $D \subseteq\{2, \ldots, m\}$.

To a partition $\mu$ and a subset $D \subseteq\{(i, j) \in \mu: i>1\}$, we associate a tuple of ribbons

$$
\boldsymbol{\nu}(\mu, D)=\left(\nu^{(1)}, \ldots, \nu^{(k)}\right),
$$

where $k=\mu_{1}$ is the number of columns of $\mu$, and $\nu^{(j)}$ has size $\mu_{j}^{\prime}$, cell contents $\left\{1,2, \ldots, \mu_{j}^{\prime}\right\}$, and descent set $\operatorname{Des}\left(\nu^{(j)}\right)=\{i:(i, j) \in D\}$.

Proposition 3.4. We have

$$
F_{\mu, D}(x ; q)=G_{\boldsymbol{\nu}(\mu, D)}(x ; q) .
$$

Proof. Let $\bigsqcup \boldsymbol{\nu}$ be the disjoint union of the ribbons $\nu^{(j)}$. Then we can identify semistandard tableaux of shape $\boldsymbol{\nu}$ with suitable functions $T: \bigsqcup \boldsymbol{\nu} \rightarrow \mathbb{Z}_{+}$. Let $\theta: \bigsqcup \boldsymbol{\nu} \rightarrow \mu$ be the bijection mapping the cell $u \in \nu^{(j)}$ with content $c(u)=i$ to the cell $(i, j) \in \mu$. Then $\theta$ maps $\nu^{(j)}$ onto the $j$-th column of $\mu$, and for any filling $\sigma: \mu \rightarrow \mathbb{Z}_{+}$, we see that $T=\sigma \circ \theta$ is a semistandard tableau if and only if $\operatorname{Des}(\sigma)=D$. Comparing the definition of inversions for a filling $\sigma$ of $\mu$ with the definition of inversions for a semistandard tableau $T \in \operatorname{SSYT}(\boldsymbol{\nu})$, we also see that $|\operatorname{Inv}(\sigma)|=\operatorname{inv}(T)$. This implies (27).

Theorem 3.1 follows immediately from Theorem 3.3 and Proposition 3.4

The symmetry theorem for LLT polynomials, Theorem 3.3 is a crucial ingredient in the proof of our main result. Its original proof in [20, 24] relies on a construction of Kashiwara, Miwa and Stern [14 in the representation theory of affine Hecke algebras. Apart from Theorem 3.3 all the results in this paper are deduced by elementary combinatorial means. To remove this one exception, we present in 10 a new, elementary proof of Theorem 3.3 .

\section{QUASISYMMETRIC FUNCTION EXPANSION AND SUPERIZATION}

Given a non-negative integer $n$ and a subset $D \subseteq\{1, \ldots, n-1\}$, Gessel's quasisymmetric function $Q_{n, D}(x)$ of degree $n$ in variables $x=x_{1}, x_{2}, \ldots$ is defined by the formula

$$
Q_{n, D}(x)=\sum_{\substack{a_{1} \leq a_{2} \leq \cdots \leq a_{n} \\ a_{i}=a_{i+1} \Rightarrow i \notin D}} x_{a_{1}} x_{a_{2}} \cdots x_{a_{n}}
$$

where the indices $a_{i}$ belong to $\mathbb{Z}_{+}$. More generally, consider a "super" alphabet

$$
\mathcal{A}=\mathbb{Z}_{+} \cup \mathbb{Z}_{-}=\{\overline{1}, 1, \overline{2}, 2, \ldots\}
$$

of positive letters $i$ and negative letters $\bar{i}$. We will use two different orderings of $\mathcal{A}$ :

$$
\begin{aligned}
& \left(\mathcal{A},<_{1}\right)=\{1<\overline{1}<2<\overline{2}<\cdots\} \\
& \left(\mathcal{A},<_{2}\right)=\{1<2<3<\cdots<\overline{3}<\overline{2}<\overline{1}\} .
\end{aligned}
$$


Fix now either of these, or any total ordering of $\mathcal{A}$. The "super" quasisymmetric function $\tilde{Q}_{n, D}(x, y)$ in variables $x=x_{1}, x_{2}, \ldots$ and $y=y_{1}, y_{2}, \ldots$ is defined by

$$
\tilde{Q}_{n, D}(x, y)=\sum_{\substack{a_{1} \leq a_{2} \leq \cdots \leq a_{n} \\ a_{i}=a_{i+1} \in \mathbb{Z}_{+} \Rightarrow i \notin D \\ a_{i}=a_{i+1} \in \mathbb{Z}_{-} \Rightarrow i \in D}} z_{a_{1}} z_{a_{2}} \cdots z_{a_{n}},
$$

where the indices $a_{i}$ belong to $\mathcal{A}$, and we set $z_{i}=x_{i}$ for $i$ positive, $z_{\bar{i}}=y_{i}$ for $\bar{i}$ negative.

Definition 4.1. The superization of a symmetric function $f(x)$ is $\tilde{f}(x, y)=$ $\omega_{Y} f[X+Y]$ (the subscript $Y$ indicating that $\omega$ acts on $f[X+Y]=f(x, y)$ considered as a symmetric function of the $y$ variables only).

Proposition $4.2(9)$. Let $f(x)$ be a symmetric function homogeneous of degree $n$, written in terms of quasisymmetric functions as

$$
f(z)=\sum_{D} c_{D} Q_{n, D}(z) .
$$

Then its superization is given by

$$
\tilde{f}(x, y)=\sum_{D} c_{D} \tilde{Q}_{n, D}(x, y)
$$

We remark that the proposition is well known and that the proof outlined in [9] works equally well for any chosen ordering of the alphabet $\mathcal{A}$.

Next we give the quasisymmetric function expansion of the polynomial $C_{\mu}(x ; q, t)$ and its superization $\tilde{C}_{\mu}(x, y ; q, t)$. Given a super alphabet $\mathcal{A}$, a super filling of $\mu$ is a function $\sigma: \mu \rightarrow \mathcal{A}$. We adapt the definitions of $\operatorname{Inv}(\sigma)$ and $\operatorname{Des}(\sigma)$ to super fillings as follows. Extend the notation $I(x, y)$ in (16) to $x, y \in \mathcal{A}$ by setting

$$
I(x, y)= \begin{cases}1 & \text { if } x>y \text { or } x=y \in \mathbb{Z}_{-}, \\ 0 & \text { if } x<y \text { or } x=y \in \mathbb{Z}_{+} .\end{cases}
$$

For cells $u$ directly above $v$ in $\mu$, we say that $\sigma(u)$ and $\sigma(v)$ form a descent if $I(\sigma(u), \sigma(v))=1$, and as before, we take $\operatorname{Des}(\sigma)$ to be the set of cells $u$ occurring as the upper cell in a descent. An inversion is a pair of entries $\sigma(u), \sigma(v)$ such that $I(\sigma(u), \sigma(v))=1$, the cells $u$ and $v$ attack each other, and $u$ precedes $v$ in the reading order. As before, $\operatorname{Inv}(\sigma)$ is the set of positions forming inversions in $\sigma$. The statistics $\operatorname{inv}(\sigma)$ and maj $(\sigma)$ are defined in terms of $\operatorname{Inv}(\sigma)$ and $\operatorname{Des}(\sigma)$ by (13), as for ordinary fillings. The definition of inversion triples and the demonstration that $\operatorname{inv}(\sigma)$ is non-negative go through verbatim with the extended definition of $I(x, y)$. Note that an ordinary filling is the special case of a super filling with only positive entries.

Define a filling $\sigma$ to be standard if it is a bijection $\sigma: \mu \cong\{1, \ldots, n\}$. Given a super filling $\sigma$, its standardization is the unique standard filling $\xi$ such that $\sigma \circ \xi^{-1}$ is weakly increasing, and for each $x \in \mathcal{A}$, the restriction of $\xi$ to $\sigma^{-1}(\{x\})$ is increasing with respect to the reading order if $x$ is positive, decreasing if $x$ is negative. An example, using the ordering $<_{1}$ in (30) on $\mathcal{A}$, is

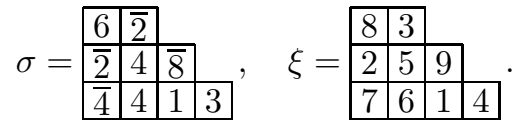


It is immediate from the definitions that $\operatorname{Inv}(\sigma)=\operatorname{Inv}(\xi), \operatorname{Des}(\sigma)=\operatorname{Des}(\xi)$, $\operatorname{inv}(\sigma)=\operatorname{inv}(\xi)$, and $\operatorname{maj}(\sigma)=\operatorname{maj}(\xi)$.

Define the reading word of a filling to be the sequence of its entries listed in the reading order. Then the reading word of a standard filling $\xi$ is a permutation of $\{1, \ldots, n\}$, where $n=|\mu|$. Let $D(\xi) \subseteq\{1, \ldots, n-1\}$ be the descent set of the inverse permutation, that is, $i \in D(\xi)$ if $\xi^{-1}(i+1)$ precedes $\xi^{-1}(i)$ in the reading order. For the example in (35), we have $D(\xi)=\{1,2,4,6,7\}$. If $\xi$ is the standardization of $\sigma$, the weakly increasing function $a=\sigma \circ \xi^{-1}:\{1, \ldots, n\} \rightarrow \mathcal{A}$ also satisfies the conditions: $a(i)=a(i+1) \in \mathbb{Z}_{+}$implies $i \notin D(\xi)$, and $a(i)=a(i+1) \in \mathbb{Z}_{-}$implies $i \in D(\xi)$. Conversely, given $\xi$ and $a$ satisfying these conditions, $\sigma=a \circ \xi$ is a super filling whose standardization is $\xi$. These observations together with Theorem 3.1 and Proposition 4.2 yield the following formulas.

Proposition 4.3. With $n=|\mu|$, the polynomial $C_{\mu}(x ; q, t)$ has the quasisymmetric function expansion given by the sum over standard fillings

$$
C_{\mu}(x ; q, t)=\sum_{\xi: \mu \cong\{1, \ldots, n\}} q^{\operatorname{inv}(\xi)} t^{\operatorname{maj}(\xi)} Q_{n, D(\xi)}(x) .
$$

Its superization $\tilde{C}_{\mu}(x, y ; q, t)=\omega_{Y} C_{\mu}[X+Y ; q, t]$ has the expansion

$$
\tilde{C}_{\mu}(x, y ; q, t)=\sum_{\xi: \mu \cong\{1, \ldots, n\}} q^{\operatorname{inv}(\xi)} t^{\operatorname{maj}(\xi)} \tilde{Q}_{n, D(\xi)}(x, y) .
$$

This last is equal to the generating function for super fillings

$$
\tilde{C}_{\mu}(x, y ; q, t)=\sum_{\sigma: \mu \rightarrow \mathcal{A}} q^{\operatorname{inv}(\sigma)} t^{\operatorname{maj}(\sigma)} z^{\sigma},
$$

where $z_{i}=x_{i}$ for $i$ positive, $z_{\bar{i}}=y_{i}$ for $\bar{i}$ negative.

\section{Proof of the Formula}

This section is devoted to the proof of Theorem 2.2 We will prove that the combinatorial expression $C_{\mu}(x ; q, t)$ in Definition2.1 satisfies the defining conditions (T1)-(T2) and (N) for $\tilde{H}_{\mu}(x ; q, t)$ displayed in (4). We will do this by introducing a sign-reversing involution on super fillings to prove each of (T1)-(T2).

Before proceeding further, we rewrite the conditions (T1)-(T2) in a more convenient form. Recall that for any plethystic alphabet $Y$ and any symmetric function $f$ homogeneous of degree $d$, we have $f[-Y]=(-1)^{d}(\omega f)[Y]$. Also recall that $\omega s_{\lambda}(x)=s_{\lambda^{\prime}}(x)$, and that transpose reverses the partial ordering on partitions: $\lambda \leq \rho \Leftrightarrow \rho^{\prime} \leq \lambda^{\prime}$. Finally, recall that the Schur and monomial bases are mutually lower triangular with respect to this ordering, i.e., $s_{\lambda} \in \mathbb{Z}\left\{m_{\rho}: \rho \leq \lambda\right\}$ and $m_{\rho} \in \mathbb{Z}\left\{s_{\lambda}: \lambda \leq \rho\right\}$. Using these facts, we see that (T1)-(T2) are equivalent to

$$
\begin{aligned}
& \tilde{H}_{\mu}[X(q-1) ; q, t]=\sum_{\rho \leq \mu^{\prime}} c_{\rho \mu}(q, t) m_{\rho}(x), \\
& \tilde{H}_{\mu}[X(t-1) ; q, t]=\sum_{\rho \leq \mu} d_{\rho \mu}(q, t) m_{\rho}(x)
\end{aligned}
$$

for suitable coefficients $c_{\rho \mu}, d_{\rho \mu}$.

Now consider condition $(N)$. Since $\left\{h_{\mu}\right\}$ and $\left\{m_{\mu}\right\}$ are dual bases relative to the Hall scalar product and since $s_{(n)}=h_{n},(N)$ is equivalent to the requirement that the coefficient of $x_{1}^{n}$ in $\tilde{H}_{\mu}(x ; q, t)$ be equal to 1 . It is immediate from the 
definition that $C_{\mu}(x ; q, t)$ satisfies this condition, since the filling $\sigma(u)=1$ for all $u$ has $\operatorname{maj}(\sigma)=\operatorname{inv}(\sigma)=0$.

To show that $C_{\mu}(x ; q, t)$ satisfies (A1)-(A2), we need combinatorial interpretations for the expansion into monomials of $C_{\mu}[X(q-1) ; q, t]$ and $C_{\mu}[X(t-1) ; q, t]$. For this we use the identities $C_{\mu}[X(q-1) ; q, t]=\tilde{C}_{\mu}(q x,-x ; q, t), C_{\mu}[X(t-1) ; q, t]=$ $\tilde{C}_{\mu}(t x,-x ; q, t)$, which follow from the general identity $f[X-Y]=\tilde{f}(x,-y)$, where $\tilde{f}(x, y)=\omega_{Y} f[X+Y]$. Applying (38), we obtain

$$
\begin{aligned}
& C_{\mu}[X(q-1) ; q, t]=\sum_{\sigma: \mu \rightarrow \mathcal{A}}(-1)^{m(\sigma)} q^{p(\sigma)+\operatorname{inv}(\sigma)} t^{\operatorname{maj}(\sigma)} x^{|\sigma|}, \\
& C_{\mu}[X(t-1) ; q, t]=\sum_{\sigma: \mu \rightarrow \mathcal{A}}(-1)^{m(\sigma)} q^{\operatorname{inv}(\sigma)} t^{p(\sigma)+\operatorname{maj}(\sigma)} x^{|\sigma|},
\end{aligned}
$$

where $m(\sigma)=\left|\left\{u: \sigma(u) \in \mathbb{Z}_{-}\right\}\right|$and $p(\sigma)=\left|\left\{u: \sigma(u) \in \mathbb{Z}_{+}\right\}\right|$are the numbers of negative and positive entries in the super filling $\sigma$, and $x^{|\sigma|}=\prod_{u \in \mu} x_{|\sigma(u)|}$. Note that these formulas are valid with $\operatorname{inv}(\sigma)$ and $\operatorname{maj}(\sigma)$ defined with respect to any chosen ordering of $\mathcal{A}$. As it turns out, the ordering $<_{1}$ in (30) is best suited to analyze (40), and $<_{2}$ to analyze (41).

5.1. Proof that $C_{\mu}(x ; q, t)$ satisfies $(A 1)$. We use the ordering $<_{1}$ on $\mathcal{A}$. We shall construct a sign-reversing, weight-preserving involution $\Psi$ on super fillings $\sigma: \mu \rightarrow \mathcal{A}$, which cancels out all terms in (40) involving $x^{\rho}$ if $\rho \not \leq \mu^{\prime}$.

If there is no pair of attacking cells $u, v$ such that $|\sigma(u)|=|\sigma(v)|$, define $\Psi \sigma=\sigma$. Otherwise, let $a$ be the smallest integer that occurs as $|\sigma(u)|=|\sigma(v)|$ for some attacking pair. Fix $v$ to be the last cell in the reading order that is part of an attacking pair with $|\sigma(u)|=|\sigma(v)|=a$, and fix $u$ to be the last cell in the reading order that attacks $v$ and has $|\sigma(u)|=a$. Now define $\Psi \sigma(w)=\sigma w$ for all $w \neq u$, and $\Psi \sigma(u)=\overline{\sigma(u)}$, i.e., applying $\Psi$ flips the sign of the entry in cell $u$. Clearly, $\Psi \Psi \sigma=\sigma$, since $a, u$ and $v$ only depend on $|\sigma|$.

Note that the indicator $I(x, y)$ in (34), when defined with respect to the ordering $<_{1}$, has the property that

$$
I(x, y)=I(x, \bar{y}) \quad \text { for all } x, y \in \mathcal{A} .
$$

Lemma 5.1. We have

$$
C_{\mu}[X(q-1) ; q, t]=\sum_{\Psi \sigma=\sigma}(-1)^{m(\sigma)} q^{p(\sigma)+\operatorname{inv}(\sigma)} t^{\operatorname{maj}(\sigma)} x^{|\sigma|} .
$$

Proof. For $\Psi \sigma \neq \sigma$, we have $m(\Psi \sigma)=m(\sigma) \pm 1$, so $\Psi$ is sign-reversing. Obviously, $x^{|\Psi \sigma|}=x^{|\sigma|}$. To prove (43), we need to show that $\Psi$ preserves the weight $q^{p(\sigma)+\operatorname{inv}(\sigma)} t^{\operatorname{maj}(\sigma)}$.

Take $a, u, v$ as in the definition of $\Psi$. Interchanging $\sigma$ and $\Psi \sigma$ if necessary, we can assume that $\sigma(u)$ is positive, i.e., that $\sigma(u)=a, \Psi \sigma(u)=\bar{a}$. We first show that $\operatorname{Des}(\Psi \sigma)=\operatorname{Des}(\sigma)$, which implies maj $(\Psi \sigma)=\operatorname{maj}(\sigma)$. For this, consider the entries (if any) directly above and below cell $u$ in $\sigma$ and in $\Psi \sigma$ :

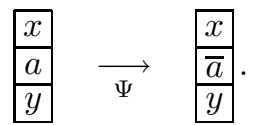

Either $x$ or $y$ may be missing, if $u$ is at the top or bottom of a column. The cell $w$ below $u$, if present, follows $v$ in the reading order and attacks $v$. By definition, $v$ is 
the last cell in the reading order that has $|\sigma(v)|=a$ and attacks another cell with the same property. Hence $|y| \neq a$. In the ordering $<_{1}$, this implies $I(a, y)=I(\bar{a}, y)$, so $u \in \operatorname{Des}(\Psi \sigma)$ if and only if $u \in \operatorname{Des}(\sigma)$. If the cell $t$ directly above $u$ is present, then (42) shows that $t \in \operatorname{Des}(\Psi \sigma)$ if and only if $t \in \operatorname{Des}(\sigma)$. Hence $\operatorname{Des}(\Psi \sigma)=\operatorname{Des}(\sigma)$, as claimed.

By assumption, $p(\Psi \sigma)=p(\sigma)-1$, so it remains to prove that $\operatorname{inv}(\Psi \sigma)=\operatorname{inv}(\sigma)+$ 1. We already have $\operatorname{Des}(\Psi \sigma)=\operatorname{Des}(\sigma)$, so we are to prove that $|\operatorname{Inv}(\Psi \sigma)|=$ $|\operatorname{Inv}(\sigma)|+1$. Now, $\{u, v\}$ belongs to $\operatorname{Inv}(\Psi \sigma)$ but not to $\operatorname{Inv}(\sigma)$, since $|\sigma(v)|=a$, and for $|y|=a$, we have $I(\bar{a}, y)=1, I(a, y)=0$. We claim that $\operatorname{Inv}(\Psi \sigma)$ and $\operatorname{Inv}(\sigma)$ are otherwise identical. Clearly, the only other inversions that might differ are of the form $\{u, w\}$, where $u$ attacks $w$. By (42), we can assume further that $w$ follows $u$ in the reading order. Moreover, we must have $|w|=a$. But then $w$ precedes $v$ in the reading order, by the definition of $v$. This contradicts the definition of $u$. The lemma is proved.

The fixed points of $\Psi$ are non-attacking fillings $\sigma: \mu \rightarrow \mathcal{A}$, characterized by the property that if $u, v \in \mu$ attack each other, then $|\sigma(u)| \neq|\sigma(v)|$. In particular, this implies that for all $x \in \mathbb{Z}_{+}$, there is at most one entry of $\sigma$ with absolute value $x$ in each row of $\mu$. Suppose $\rho$ is a partition and $x^{|\sigma|}=x^{\rho}=x_{1}^{\rho_{1}} x_{2}^{\rho_{2}} \cdots x_{l}^{\rho_{l}}$ for some non-attacking filling $\sigma$. Then $\rho_{1}+\cdots+\rho_{j}$ is the total number of entries in $\sigma$ with absolute value at most $j$. By the preceding observation, this cannot exceed $\sum_{i} \min \left(\mu_{i}, j\right)=\mu_{1}^{\prime}+\cdots+\mu_{j}^{\prime}$. Hence $\rho \leq \mu^{\prime}$, proving that $C_{\mu}(x ; q, t)$ satisfies $(A 1)$.

5.2. Proof that $C_{\mu}(x ; q, t)$ satisfies (A2). We use the ordering $<_{2}$ on $\mathcal{A}$. We shall construct a sign-reversing, weight-preserving involution $\Phi$ on super fillings $\sigma: \mu \rightarrow \mathcal{A}$, which cancels out all terms in (41) involving $x^{\rho}$ if $\rho \not \leq \mu$.

If $|\sigma(u)| \geq i$ for all cells $u=(i, j) \in \mu$, define $\Phi \sigma=\sigma$. Otherwise, let $a$ be the smallest integer which occurs as $|\sigma(u)|<i$ for some $u=(i, j)$. Let $u$ be the first cell in the reading order with $|\sigma(u)|=a$; note that the row coordinate $i$ is maximal for this cell, so $a<i$. Define $\Phi \sigma(w)=\sigma(w)$ for all $w \neq u$, and $\Phi \sigma(u)=\overline{\sigma(u)}$, so applying $\Phi$ flips the sign of the entry in cell $u$. Clearly, $\Phi \Phi \sigma=\sigma$, since $a$ and $u$ depend only on $|\sigma|$.

Lemma 5.2. We have

$$
C_{\mu}[X(t-1) ; q, t]=\sum_{\Phi \sigma=\sigma}(-1)^{m(\sigma)} q^{\operatorname{inv}(\sigma)} t^{p(\sigma)+\operatorname{maj}(\sigma)} x^{|\sigma|} .
$$

Proof. As in the proof of Lemma 5.1, $\Phi$ is sign-reversing and preserves $x^{|\sigma|}$. Take $a, u$ as in the definition of $\Phi$. We may assume that $\sigma(u)=a, \Phi \sigma(u)=\bar{a}$. Then we are to prove that $\operatorname{inv}(\Phi \sigma)=\operatorname{inv}(\sigma)$ and $\operatorname{maj}(\Phi \sigma)=\operatorname{maj}(\sigma)+1$. Note that by construction, $u$ is in row $i$ with $i>a$, so $u$ is not in the bottom row of $\mu$.

For maj $(\Phi \sigma)$, consider the entries directly above and below cell $u$ :

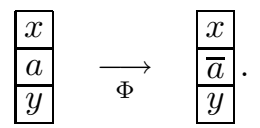

Here $x$ may be missing, but $y$ is always present. Moreover, $|y| \geq a$, since $|y|<a$ would imply $|y|<i-1$, and since $y$ is in row $i-1$, this would contradict the choice of $a$. In the ordering $<_{2}$, for $|y| \geq a$, we have $I(a, y)=0, I(\bar{a}, y)=1$. Hence $u \in \operatorname{Des}(\Phi \sigma), u \notin \operatorname{Des}(\sigma)$. 
Suppose there is a cell $t$ directly above $u$ in $\mu$, with $\sigma(t)=x$. Then $|x| \neq a$, by the choice of $u$. If $|x|<a$, then $|x|<i+1$, contradicting the choice of $a$. Hence $|x|>a$. In the ordering $<_{2}$, this implies $I(x, a)=1, I(x, \bar{a})=0$, so $t \in \operatorname{Des}(\sigma)$, $t \notin \operatorname{Des}(\Phi \sigma)$. Clearly $\operatorname{Des}(\Phi \sigma)$ and $\operatorname{Des}(\sigma)$ differ only in the cells $u$ and $t$. Since $\operatorname{leg}(u)=\operatorname{leg}(t)+1$, this gives $\operatorname{maj}(\Phi \sigma)=\operatorname{maj}(\sigma)+1$. Alternatively, if $u$ is the top cell in its column, $\operatorname{Des}(\Phi \sigma)$ and $\operatorname{Des}(\sigma)$ differ only in cell $u$, and $\operatorname{leg}(u)=0$, so we have $\operatorname{maj}(\Phi \sigma)=\operatorname{maj}(\sigma)+1$ in this case too.

Recall from the discussion at the end of 92 that $\operatorname{inv}(\sigma)$ is the number of inversions in row 1 plus the number of inversion triples in $\sigma$. Since $\sigma$ and $\Phi \sigma$ are identical in row 1 , they have the same inversions there. To complete the proof, we verify that $\sigma$ and $\Phi \sigma$ have the same inversion triples. A triple that might differ must include the cell $u$. There are three cases.

Case I: $u$ is the bottom cell in the triple, so we have

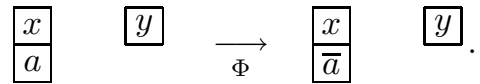

Then $|x|,|y| \neq a$, by the choice of $u$, and $|x|,|y| \nless a$, by the choice of $a$. Hence $|x|,|y|>a$ and $a<_{2} x, y<_{2} \bar{a}$. In $\sigma$ we have $I(x, a)=I(y, a)=1$, while in $\Phi \sigma$ we have $I(x, \bar{a})=I(y, \bar{a})=0$. In both $\sigma$ and $\Phi \sigma$, this triple is an inversion triple if and only if $I(x, y)=1$.

Case II: $u$ is the upper right cell in the triple, so we have

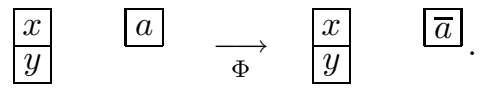

The choice of $a$ and $u$ implies $|x|>a$ and $|y| \geq a$, so $a<_{2} x<_{2} \bar{a}$ and $a \leq_{2} y \leq_{2} \bar{a}$. In $\sigma$, we have $I(x, a)=1, I(a, y)=0$, while in $\Phi \sigma$, we have $I(x, \bar{a})=0, I(\bar{a}, y)=1$. In both $\sigma$ and $\Phi \sigma$, this is an inversion triple if and only if $I(x, y)=0$.

Case III: $u$ is the upper left cell in the triple, so we have

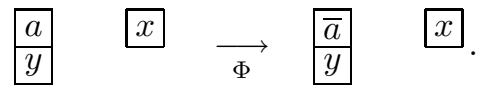

We deduce that $|x|,|y| \geq a$, so $a \leq_{2} x, y \leq_{2} \bar{a}$. In $\sigma$, we have $I(a, x)=I(a, y)=0$, while in $\Phi \sigma$, we have $I(\bar{a}, x)=I(\bar{a}, y)=1$. In both $\sigma$ and $\Phi \sigma$, this is an inversion triple if and only if $I(x, y)=1$.

If $\sigma=\Phi \sigma$ is a fixed point, then all entries $x$ with $|x| \leq j$ occur in rows 1 through $j$. If $\rho$ is a partition and $x^{|\sigma|}=x^{\rho}$, we therefore have $\rho_{1}+\cdots+\rho_{j} \leq \mu_{1}+\cdots+\mu_{j}$ for all $j$, that is, $\rho \leq \mu$. This proves that $C_{\mu}(x ; q, t)$ satisfies (A2) and completes the proof of Theorem 2.2 .

\section{MACDONALD SPECIALIZATION}

In this and the next two sections we discuss some previously known results from the theory of Macdonald and Jack polynomials that can be deduced directly from Theorem 2.2 Our first example is the following proposition, equivalent to an identity of Macdonald [26, Ch. VI (8.8)].

Proposition 6.1. The coefficient of $(-u)^{d}$ in $\tilde{H}_{\mu}[1-u ; q, t]$ is equal to $e_{d}\left[B_{\mu}\right]$, where

$$
B_{\mu}=\sum_{(i, j) \in \mu} t^{i-1} q^{j-1}
$$


Remark. The proposition is equivalent to the formula $\tilde{K}_{\lambda \mu}(q, t)=e_{d}\left[B_{\mu}-1\right]$ for hook shapes $\lambda=\left(n-d, 1^{d}\right)$.

Proof. From formula (38) we see that the coefficient in question is the sum of $q^{\operatorname{inv}(\sigma)} t^{\operatorname{maj}(\sigma)}$ over super fillings $\sigma$ with $n-d$ entries equal to 1 and $d$ entries equal to $\overline{1}$. Use an ordering in which $1<\overline{1}$. Then $u \in \operatorname{Des}(\sigma)$ if and only if $\sigma(u)=\overline{1}$ and $u$ is not in row 1 . Furthermore, each such $u$ forms an inversion with every cell to its right in the same row and with every cell to its left in the row below. Subtracting $\operatorname{arm}(u)$, the contribution to $\operatorname{inv}(\sigma)$ from $u=(i, j) \in \operatorname{Des}(\sigma)$ is $j-1$. The contribution to $\operatorname{maj}(\sigma)$ from $u$ is $\operatorname{leg}(u)+1$.

For $u$ in row 1 with $\sigma(u)=\overline{1}$ we get an inversion between $u$ and every cell to its right. These observations show that if for $u=(i, j)$, we define

$$
L(u)= \begin{cases}t^{\operatorname{leg}(u)+1} q^{j-1} & \text { if } i \neq 1, \\ q^{\operatorname{arm}(u)} & \text { if } i=1,\end{cases}
$$

then $q^{\operatorname{inv}(\sigma)} t^{\operatorname{maj}(\sigma)}=\prod_{\sigma(u)=\overline{1}} L(u)$. Summing over fillings with $n-d 1$ 's and $d \overline{1}$ 's, the result follows, once we verify that

$$
\sum_{u \in \mu} L(u)=B_{\mu} .
$$

Consider the figure below, in which the entries $q^{j-1} t^{i-1}$ in the first diagram sum to $B_{\mu}$, and the entries in the second diagram are $L(u)$.

\begin{tabular}{|c|c|}
\hline$t$ & $q t$ \\
\hline$t^{2}$ & $q t^{2}$ \\
\hline$q^{2}$ & $q$ \\
\hline
\end{tabular}

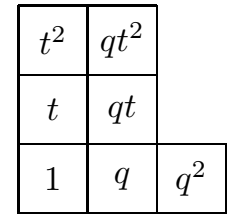

In this example and in general, row 1 in the second diagram is the reverse of row 1 in the first diagram, and except for row 1, each column in the second diagram is the reverse of the corresponding column in the first diagram. This proves (52).

\section{Cocharge specialization}

Next we show that the celebrated charge formula of Lascoux and Schützenberger 21], which expresses the Hall-Littlewood polynomials in terms of Schur functions, arises naturally as a corollary to Theorem 2.2

Proposition 7.1. We have

$$
\tilde{H}_{\mu}(x ; 0, t)=\sum_{\lambda}\left(\sum_{T \in \operatorname{SSYT}(\lambda, \mu)} t^{\mathrm{cc}(T)}\right) s_{\lambda}(x),
$$

where $\operatorname{cc}(T)$ is the cocharge of $T$. The sum is over semistandard tableaux $T$ of shape $\lambda$ and content $\mu$, i.e., such that the multiset of entries in $T$ is $\left\{1^{\mu_{1}}, 2^{\mu_{2}}, \ldots, l^{\mu_{l}}\right\}$.

Before proving the proposition, let us recall the definition of cocharge. Let $\mu$ be a partition of $n$, and let $w=w_{1} \cdots w_{n}$ be a word whose multiset of letters is $\left\{1^{\mu_{1}}, 2^{\mu_{2}}, \ldots, l^{\mu_{l}}\right\}$. Such a word $w$ is said to have partition content. One defines cocharge in terms of words, then extends the definition to tableaux $T$ with partition content by setting $\operatorname{cc}(T)=\operatorname{cc}(w)$, where $w$ is the reading word of $T$ (the sequence of its entries listed in the reading order). 
If $w$ is a permutation, i.e., if $\mu=\left(1^{n}\right)$, then

$$
\operatorname{cc}(w) \underset{\text { def }}{=} \operatorname{comaj}\left(w^{-1}\right)=\sum_{k \in D\left(w^{-1}\right)}(n-k),
$$

where $D\left(w^{-1}\right)=\left\{i: w^{-1}(i)>w^{-1}(i+1)\right\}$ is the descent set of the inverse permutation.

In the general case, we first extract a subword $y$ of $w$ as follows. Let $k_{1}=$ $\max \left\{k: w_{k}=1\right\}$ be the position of the rightmost 1 in $w$, and define $k_{2}, \ldots, k_{l}$ inductively by $k_{i}=\max \left\{k<k_{i-1}: w_{k}=i\right\}$ if this set is non-empty, or $k_{i}=$ $\max \left\{k: w_{k}=i\right\}$, otherwise. In less formal terms, one can think of scanning the word from right to left, returning to the right when necessary, seeking entries $w_{k_{1}}=1, w_{k_{2}}=2, \ldots, w_{k_{l}}=l$ in succession. Let $S=\left\{k_{1}, \ldots, k_{l}\right\}$, let $y$ be the subword of $w$ indexed by $S$, and let $z$ be the subword of $w$ indexed by the complement of $S$. Then $y$ is a permutation of $\{1, \ldots, l\}, z$ again has partition content, and the cocharge is defined inductively as $\operatorname{cc}(w)=\operatorname{cc}(y)+\operatorname{cc}(z)$.

Proof of Proposition 7.1. Since $C_{\mu}(x ; 0, t)$ enumerates fillings with $\operatorname{inv}(\sigma)=0$, we begin by describing their structure. Let $l$ be the length of $\mu$ and for each $i=1, \ldots, l$, fix a multiset $M_{i}$ of $\mu_{i}$ positive integers. Consider those fillings $\sigma$ in which $M_{i}$ is the multiset of entries in row $i$. By a lemma in [8, there is a unique such $\sigma$ with $\operatorname{inv}(\sigma)=0$. We can also see directly how to uniquely construct the required $\sigma$, by observing that $\operatorname{inv}(\sigma)=0$ if and only if

(i) $\sigma$ is non-decreasing in row 1 ; and

(ii) for every cell $u$ not in row 1 , if $v$ is the cell directly below $u$ and $S$ is the set consisting of $u$ and the cells to its right in the same row, then $\sigma(u) \leq \sigma(v)$ implies that $x \leq \sigma(v)$ for all $x \in \sigma(S)$, and $\sigma(u)=\min \sigma(S)$, while $\sigma(u)>\sigma(v)$ implies that $\sigma(u)=\min \{x \in \sigma(S): x>\sigma(v)\}$.

Hence the entries of $\sigma$ in row 1 must be the elements of $M_{1}$ in non-decreasing order. Once rows 1 through $i-1$ have been constructed, the entries $\sigma(u)$ in row $i$ are determined one by one, from left to right, as follows. Let $v$ be the cell directly below $u$. If $M_{i}$ contains an unused element $x>\sigma(v)$, then $\sigma(u)$ is the smallest such $x$; otherwise $\sigma(u)$ is the smallest $x \in M_{i}$ not yet used. For example, if $\mu=(5,5,3,1)$, $M_{1}=\{1,1,3,6,7\}, M_{2}=\{1,2,4,4,5\}, M_{3}=\{1,2,3\}$, and $M_{4}=\{2\}$, then $\sigma$ is the filling shown below.

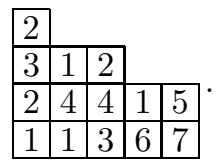

Given a filling $\sigma: \mu \rightarrow \mathbb{Z}_{+}$, let $u_{1}=\left(i_{1}, j_{1}\right), \ldots, u_{n}=\left(i_{n}, j_{n}\right)$ be the ordering of the cells of $\mu$ such that $\sigma\left(u_{1}\right) \geq \cdots \geq \sigma\left(u_{n}\right)$, and for each constant segment $\sigma\left(u_{j}\right)=\cdots=\sigma\left(u_{k}\right)$, the cells $u_{j}, \ldots, u_{k}$ are in decreasing reading order. We define the cocharge word $\mathrm{cw}(\sigma)=i_{1} i_{2} \cdots i_{n}$ to be the list of row indices of the cells $u_{k}$ in this order. Note that $\mathrm{cw}(\sigma)$ has partition content $\mu$. For the filling $\sigma$ shown in $(56), \mathrm{cw}(\sigma)=11222132341123$.

We claim that if $\operatorname{inv}(\sigma)=0$, then $\operatorname{maj}(\sigma)=\operatorname{cc}(\operatorname{cw}(\sigma))$. To see this, consider the symbols $i_{k_{1}}=1, \ldots, i_{k_{l}}=l$ in $\mathrm{cw}(\sigma)$ corresponding to the cells $u_{k_{1}}=$ $(1,1), \ldots, u_{k_{l}}=(l, 1)$ in the first column of $\mu$. The fact that $\sigma((1,1))$ is the smallest entry in row 1 implies that $i_{k_{1}}$ is the rightmost $1 \mathrm{in} \operatorname{cw}(\sigma)$. For $i>1, \sigma((i, 1))$ is 
the smallest entry greater than $\sigma((i-1,1))$ in row $i$, if one exists; otherwise $\sigma((i, 1))$ is the smallest entry in row $i$ entirely. This implies that $i_{k_{i}}$ is the rightmost $i$ to the left of $i_{k_{i-1}}$ in $\mathrm{cw}(\sigma)$, if one exists; otherwise $i_{k_{i}}$ is the rightmost $i$ entirely. It follows that the subword $y$ in the definition of $\operatorname{cc}(\operatorname{cw}(\sigma))$ consists of $i_{k_{1}}$ through $i_{k_{l}}$. Moreover, it is clear that the descents in the first column of $\sigma$ match the descent set of the permutation $y^{-1}$, and therefore

$$
\operatorname{cc}(y)=\sum_{u=(i, 1) \in \operatorname{Des}(\sigma)}(\operatorname{leg}(u)+1) .
$$

The complementary subword $z$ of $\operatorname{cw}(\sigma)$ is just $\operatorname{cw}\left(\sigma_{1}\right)$, where $\sigma_{1}$ is the restriction of $\sigma$ to the diagram obtained by deleting the first column of $u$. We again have $\operatorname{inv}\left(\sigma_{1}\right)=0$, so the claim follows by induction.

It is known (and easy to prove using the Knuth relations; see [22, [27, Ex. 1.7.6]) that $\operatorname{cc}(w)$ is an invariant of the plactic monoid, i.e., if $P(w)$ denotes the RSK insertion tableau of $w$, then $\operatorname{cc}(w)=\operatorname{cc}(P(w))$ for every word $w$ with partition content. Let $M(\sigma)$ be the multiset of pairs $(\sigma(u), i)$, where $u=(i, j) \in \mu$. Giving $M(\sigma)$ is equivalent to giving the multisets $M_{i}$ of entries in each row. For $\mu$ fixed, $\sigma \mapsto M(\sigma)$ is therefore a bijection from fillings with $\operatorname{inv}(\sigma)=0$ to multisubsets of $\mathbb{Z}_{+} \times \mathbb{Z}_{+}$such that the projection of $M(\sigma)$ on the second index is $\left\{1^{\mu_{1}}, \ldots, l^{\mu_{l}}\right\}$. Applying RSK to $M(\sigma)$, using the reverse ordering of $\mathbb{Z}_{+}$on the first index, yields a pair $(P(\sigma), Q(\sigma))$ of semistandard tableau of the same shape, say $\lambda$. The use of the reverse ordering on the first index means that when $M(\sigma)$ is written in lexicographically non-decreasing order, the second indices form the cocharge word $\mathrm{cw}(\sigma)$. Hence $P(\sigma)=P(\operatorname{cw}(\sigma))$. By construction, $x^{\sigma}=x^{Q(\sigma)}$. Since $\sigma \mapsto(P(\sigma), Q(\sigma))$ is a bijection from fillings $\sigma$ of $\mu$ satisfying $\operatorname{inv}(\sigma)=0$ to pairs $(P, Q)$ of semistandard tableaux of the same shape, such that $P$ has content $\mu$, we deduce that

$$
\begin{aligned}
\tilde{H}_{\mu}(x ; 0, t) & =\sum_{\operatorname{inv}(\sigma)=0} t^{\operatorname{maj}(\sigma)} x^{\sigma} \\
& =\sum_{\lambda}\left(\sum_{P \in \operatorname{SSYT}(\lambda, \mu)} t^{\operatorname{cc}(P)}\right)\left(\sum_{Q \in \operatorname{SSYT}(\lambda)} x^{Q}\right),
\end{aligned}
$$

which is the same as (54).

Remark. Besides being somewhat easier than the original proof outlined in 22,22 and completed in [1] (see also [2]), our proof of Proposition 7.1 has the virtue that the rather intricate definition of cocharge emerges naturally from simpler concepts. Namely, $\operatorname{cc}(w)$ is just a way of expressing $\operatorname{maj}(\sigma)$ for fillings $\sigma$ such that $\operatorname{inv}(\sigma)=0$ and $\mathrm{cw}(\sigma)=w$.

\section{JACK SPECIALIZATION}

In this section we use Theorem 2.2 and Lemma 5.1 to obtain a new formula for the monomial expansion of Macdonald's integral form symmetric functions $J_{\mu}(x ; q, t)$, defined in [26, Ch. VI.8]. As a corollary we recover the monomial expansion of Knop and Sahi for Jack symmetric functions.

Recall from the end of $\$ 5.1$ that the fixed fillings $\Psi \sigma=\sigma$ in (43) are the nonattacking super fillings, in which $|\sigma(u)| \neq|\sigma(v)|$ for cells $u, v$ that attack each other. As in (43), $p(\sigma)$ and $m(\sigma)$ denote the number of positive and negative entries $\sigma$. We use the ordering $<_{1}$ on the super alphabet $\mathcal{A}$. 
Fix $n=|\mu|$, and define (using conflicting but standard notation)

$$
n(\mu)=\frac{\overline{d e f}}{\sum_{i}}(i-1) \mu_{i} .
$$

The relationship between $J_{\mu}(x ; q, t)$ and $\tilde{H}_{\mu}(x ; q, t)$ is given by

$$
\begin{aligned}
J_{\mu}(X ; q, t) & =t^{n(\mu)} \tilde{H}_{\mu}\left[X(1-t) ; q, t^{-1}\right] \\
& =t^{n(\mu)+n} \tilde{H}_{\mu}\left[X\left(t^{-1}-1\right) ; q, t^{-1}\right] \\
& =t^{n(\mu)+n} \tilde{H}_{\mu^{\prime}}\left[X\left(t^{-1}-1\right) ; t^{-1}, q\right],
\end{aligned}
$$

using the identity $\tilde{H}_{\mu}(x ; q, t)=\tilde{H}_{\mu^{\prime}}(x ; t, q)$, which is equivalent to [26] Ch. VI, (8.6)]. Originally, (60) was the definition of $\tilde{H}_{\mu}(x ; q, t)$. From our present point of view, (601) follows by reversing the derivation of the axiomatic characterization (4) from the original definition. Theorem 2.2 and Lemma 5.1 yield

$$
J_{\mu}(x ; q, t)=t^{n(\mu)+n} \sum_{\substack{\sigma: \mu^{\prime} \rightarrow \mathcal{A} \\ \Psi \sigma=\sigma}}(-1)^{m(\sigma)} t^{-p(\sigma)-\operatorname{inv}(\sigma)} q^{\operatorname{maj}(\sigma)} x^{|\sigma|},
$$

where the sum is over non-attacking super fillings of $\mu^{\prime}$.

For any cell $u=(i, j)$ not in the first row of $\mu$, denote the cell $v=(i-1, j)$ directly below $u$ by $\mathrm{d}(u)$. Define the absolute inversion number $\operatorname{ainv}(\sigma)$ to be the number of inversion triples $(u, v, w)$ in which the numbers $|\sigma(u)|,|\sigma(v)|,|\sigma(w)|$ are all distinct, plus the number of inversions in row 1 (necessarily with $|\sigma(u)| \neq|\sigma(v)|$, by the non-attacking property). With the ordering $<_{1}$, we see that $\operatorname{ainv}(\sigma)=\operatorname{ainv}(|\sigma|)$. Similarly, define the absolute major index

$$
\operatorname{amaj}(\sigma)=\sum_{\substack{u \in \operatorname{Des}(\sigma) \\|\sigma(u)|>|\sigma(\mathrm{d}(u))|}}(\operatorname{leg}(u)+1)
$$

to be the contribution to maj $(\sigma)$ involving descents between entries which differ in absolute value. In a positive filling, these are all the descents, so amaj $(\sigma)=$ amaj $(|\sigma|)=\operatorname{maj}(|\sigma|)$. Now define the signed inversion number and signed major index to make up the difference:

$$
\operatorname{sinv}(\sigma)=\operatorname{inv}(\sigma)-\operatorname{ainv}(\sigma) ; \quad \operatorname{smaj}(\sigma)=\operatorname{maj}(\sigma)-\operatorname{amaj}(\sigma) .
$$

Given a non-attacking positive filling $\tau$ of $\mu^{\prime}$, we now derive a formula for the part of the sum in (63) corresponding to those $\sigma$ with $|\sigma|=\tau$. Note that every such $\sigma$ is automatically non-attacking. We have

$$
\begin{aligned}
t^{n(\mu)+n} \sum_{|\sigma|=\tau} & (-1)^{m(\sigma)} t^{-p(\sigma)-\operatorname{inv}(\sigma)} q^{\operatorname{maj}(\sigma)} x^{|\sigma|} \\
& =t^{n(\mu)+n-\operatorname{ainv}(\tau)} q^{\operatorname{maj}(\tau)} x^{\tau} \sum_{|\sigma|=\tau}(-1)^{m(\sigma)} t^{-p(\sigma)-\operatorname{sinv}(\sigma)} q^{\operatorname{smaj}(\sigma)} .
\end{aligned}
$$

Consider a triple $(u, v, w)$ in $\mu^{\prime}$, with $v=\mathrm{d}(u)$. For this triple to contribute to $\operatorname{sinv}(\sigma)$, we must have at least two of the numbers $\tau(u), \tau(v), \tau(w)$ equal to each other. Since $\tau$ is non-attacking, this forces $\tau(u)=\tau(v) \neq \tau(w)$, and one checks that this is an inversion triple if and only if $\sigma(u) \in \mathbb{Z}_{+}$. Also, a cell $u$ not in row 1 belongs to $\operatorname{Des}(\sigma)$ but not to $\operatorname{Des}(\tau)$, and so it contributes to smaj $(\sigma)$ if and only if $\tau(u)=\tau(\mathrm{d}(u))$ and $\sigma(u) \in \mathbb{Z}_{-}$. 
It follows that for each cell $u \in \mu^{\prime}$ with $\tau(u)=\tau(\mathrm{d}(u))$, to calculate its contribution to the sum on the right-hand side of (66), we can weight a negative entry in $u$ by $-q^{\operatorname{leg}(u)+1}$ and a positive entry by $t^{-\operatorname{arm}(u)-1}$. For each cell $u$ such that $\tau(u) \neq \tau(\mathrm{d}(u))$, including $u$ in the bottom row, we weight a negative entry by -1 and a positive entry by $t^{-1}$. Thus (66) is equal to

$$
t^{n(\mu)+n-\operatorname{ainv}(\tau)} q^{\operatorname{maj}(\tau)} x^{\tau} \prod_{\substack{u, \mathrm{~d}(u) \in \mu^{\prime} \\ \tau(u)=\tau(\mathrm{d}(u))}}\left(t^{-\operatorname{arm}(u)-1}-q^{\operatorname{leg}(u)+1}\right) \prod_{\substack{u \in \mu^{\prime} \\ \tau(u) \neq \tau(\mathrm{d}(u))}}\left(t^{-1}-1\right) .
$$

Using (67) and the fact that for positive, non-attacking $\tau$,

$$
\operatorname{inv}(\tau)=\operatorname{ainv}(\tau)+\sum_{\substack{u, \mathrm{~d}(u) \in \mu^{\prime} \\ \tau(u)=\tau(\mathrm{d}(u))}} \operatorname{arm}(u),
$$

we obtain the following.

Proposition 8.1. For any partition $\mu$,

$$
J_{\mu}(X ; q, t)=\sum_{\substack{\tau: \mu^{\prime} \rightarrow \mathbb{Z}_{+} \\ \text {non-attacking }}} q^{\operatorname{maj}(\tau)} t^{n(\mu)-\operatorname{inv}(\tau)} x^{\tau}
$$

where the cells $u$ in the bottom row of $\mu^{\prime}$ are included in the last factor.

The integral form Jack polynomials are defined [26, Ch. VI (10.23)] by

$$
J_{\mu}^{(\alpha)}(x)=\lim _{t \rightarrow 1} \frac{J_{\mu}\left(X ; t^{\alpha}, t\right)}{(1-t)^{|\mu|}} .
$$

By setting $q=t^{\alpha}$ in Proposition 8.1 and letting $t \rightarrow 1$, we recover the following formula of Knop and Sahi [17]:

$$
J_{\mu}^{(\alpha)}(x)=\sum_{\substack{\tau: \mu^{\prime} \rightarrow \mathbb{Z}_{+} \\ \text {non-attacking }}} x^{\tau} \prod_{\substack{u \in \mu^{\prime} \\ \tau(u)=\tau(\mathrm{d}(u))}}(\alpha(\operatorname{leg}(u)+1)+\operatorname{arm}(u)+1) .
$$

\section{TWO-COLUMN CASE}

In the case where $\mu$ has only two columns, we can derive from Theorem $2.2 \mathrm{a}$ new combinatorial rule for the coefficients $\tilde{K}_{\lambda \mu}(q, t)$ in the expansion of $\tilde{H}_{\mu}(x ; q, t)$ in terms of Schur functions $s_{\lambda}(x)$.

Definition 9.1. A word $w \in \mathbb{Z}_{+}^{n}$ is Yamanouchi if each of its final segments $w_{k} w_{k+1} \cdots w_{n}$ has partition content (as defined in $\$$ /7after Proposition 7.1 ). Denote by $\operatorname{Yam}(\lambda)$ the set of Yamanouchi words with content $\left\{1^{\lambda_{1}}, \ldots, l^{\lambda_{l}}\right\}$.

Readers accustomed to English partition notation may be more familiar with the term lattice permutation. A lattice permutation is the reverse of a Yamanouchi word. 
Proposition 9.2. When $\mu_{1} \leq 2$, the coefficients in the Schur function expansion $\tilde{H}_{\mu}(x ; q, t)=\sum_{\lambda} \tilde{K}_{\lambda \mu}(q, t) s_{\lambda}(x)$ are given by

$$
\tilde{K}_{\lambda \mu}(q, t)=\sum_{\substack{\sigma: \mu \rightarrow \mathbb{Z}_{+} \\ w(\sigma) \in \operatorname{Yam}(\lambda)}} q^{\operatorname{inv}(\sigma)} t^{\operatorname{maj}(\sigma)},
$$

where $w(\sigma)$ is the reading word of $\sigma$.

When $\mu_{1} \leq 2$, Proposition 3.4 expresses $\tilde{H}_{\mu}(x ; q, t)$ in terms of LLT polynomials $G_{\boldsymbol{\nu}}(x ; q)$ in which $k=2$, i.e., $\boldsymbol{\nu}=\left(\nu^{(1)}, \nu^{(2)}\right)$. In the original formulation of Lascoux, Leclerc and Thibon [20, these LLT polynomials are domino tableau generating functions. Carré and Leclerc [3] gave a combinatorial rule for the coefficient of a Schur function in a domino LLT polynomial and outlined a proof, which was completed by van Leeuwen 31 .

Using van Leeuwen's reformulation [31, Prop. 3.1.4] of Carré and Leclerc's Yamanouchi property for domino tableaux, it is possible to show that it corresponds via the equivalences in [9] to the property that a tableau $T \in \operatorname{SSYT}(\boldsymbol{\nu})$ has Yamanouchi content reading word. Then one can deduce Proposition 9.2 from [31, Prop. 4.2.1 and Thm. 4.2.2]. However, it is complicated to trace this through in detail, besides which the proofs of the results in 31 are also complicated. It is more convenient to prove Proposition 9.2 directly by using the relationship between crystals of type $A$ and the RSK algorithm. We should remark that this is also van Leeuwen's approach (he calls the crystal operators "coplactic operations"), but it is simpler when we avoid using domino tableaux as an intermediate step.

We take as known the theory of the RSK algorithm and jeu-de-taquin, as presented for instance in [30, Ch. 7 and Appendix A1].

Definition 9.3. Let $M$ be the set of all monomials $x^{m}=x_{1}^{m_{1}} x_{2}^{m_{2}} \cdots$ in the variables $x$. A crystal (of type $A$ ) is a set $B$ equipped with a weight function wt: $B \rightarrow M$ and operators $E_{i}, F_{i}: B \rightarrow B \cup\{0\}$ for $i=1,2, \ldots$, such that

(i) $E_{i} a=b$ if and only if $F_{i} b=a$, for all $a, b \in B$, and

(ii) if $E_{i} a=b$, then $\operatorname{wt}(b)=\left(x_{i} / x_{i+1}\right) \operatorname{wt}(a)$.

The crystal $B$ is connected if the graph with vertex set $B$ and edge set $\{\{a, b\}$ : $b=E_{i} a$ for some $\left.i\right\}$ is connected.

A homomorphism between crystals is a map $\phi: B \rightarrow B^{\prime}$ such that $E_{i} \phi(b)=0$ if $E_{i} b=0, F_{i} \phi(b)=0$ if $F_{i} b=0, E_{i} \phi(a)=\phi(b)$ if $E_{i} a=b$, and $\operatorname{wt}(\phi(b))=\operatorname{wt}(b)$, for all $a, b \in B$.

An element $b \in B$ is maximal if $E_{i} b=0$ for all $i$.

The set $B=\mathbb{Z}_{+}^{n}$ of words $w=w_{1} \ldots w_{n}$ comes with a standard crystal structure. The weight function is $\operatorname{wt}(w)=x^{w}=\prod_{i} x_{w_{i}}$. For each $i$, let $y$ be the subword of $w$ consisting of letters $w_{k} \in\{i, i+1\}$. For simplicity, take $i=1$. In $y$, regard 2's as left parentheses and 1's as right parentheses. Let $z$ be the subword of $y$ that remains after repeatedly deleting all closed pairs of parentheses 21 . Then $z$ has the form $11 \cdots 122 \cdots 2$. If $z$ is all 1 's, then $E_{1} w=0$. If $z$ is all 2's, then $F_{1} w=0$. Otherwise, $E_{1} w$ is the result of changing the first 2 in $z$ to a 1 , and $F_{1} w$ is the result of changing the last 1 in $z$ to a 2 . The operators $E_{i}$ and $F_{i}$ are defined similarly. For example,

$$
342233132124 \underset{E_{2}}{\longrightarrow} 342223132124,
$$

the subword $y$ being 32233322 and the first unmatched 3 being the second 3 in $y$. 
The maximal elements in the crystal $\mathbb{Z}_{+}^{n}$ are precisely the Yamanouchi words. Note that the operator $E_{i}$ decreases the sum of the letters in $w$ by 1 . Hence every word can be reduced to a Yamanouchi word by applying a finite sequence of operators $E_{i}$.

More general crystals associated with root systems have been defined by Kashiwara 13 in connection with crystal bases, but the combinatorial crystal structure for type $A$ was known much earlier to Lascoux and Schützenberger [23]. The following lemmas are essentially due to them.

Lemma 9.4. Let $R(w)$ (the rectification of $w$ ) be the reading word of the $R S K$ insertion tableau $P(w)$. Then $R: \mathbb{Z}_{+}^{n} \rightarrow \mathbb{Z}_{+}^{n}$ is a crystal homomorphism. Moreover, the crystal operators $E_{i}, F_{i}$ on $\mathbb{Z}_{+}^{n}$ preserve the $R S K$ recording tableau $Q(w)$.

Proof. One checks easily that for any skew shape $\nu$, the set of reading words $w(T)$ for $T \in \operatorname{SSYT}(\nu)$ is closed under the crystal operators and that these operators commute with jeu-de-taquin. Jeu-de-taquin transforms the totally disconnected tableau with reading word $w$ to the RSK insertion tableau $P(w)$ with reading word $R(w)$. This implies that $R$ is a crystal homomorphism. Moreover, when the jeu-detaquin steps are performed in an order which simulates the RSK insertion algorithm as in [29], the sequence of intermediate shapes produced determines the recording tableau $Q(w)$. Hence the crystal operators do not change $Q(w)$.

Lemma 9.5. Among words with a given $R S K$ recording tableau $Q$ there is a unique Yamanouchi word $w$. The content of $w$ is equal to the shape $\lambda$ of $Q$.

Proof. Jeu-de-taquin preserves the Yamanouchi property, so this reduces to the facts that for each $\lambda$ there is a unique tableau $T \in \operatorname{SSYT}(\lambda)$ whose reading word $w(T)$ is Yamanouchi and that this $w(T)$ has content $\lambda$.

Corollary 9.6. For each standard tableau $Q$ of size $n$, the set of words $w$ with $R S K$ recording tableau equal to $Q$ is a connected component of the crystal $\mathbb{Z}_{+}^{n}$.

Proof. Lemma 9.4 implies that the recording tableau $Q$ is constant on connected components. Given two words $w, w^{\prime}$ with the same recording tableau $Q$, we can apply some sequence of operators $E_{i}$ to reduce them to Yamanouchi words $v, v^{\prime}$. Then Lemma 9.5 shows that $v=v^{\prime}$.

Proposition 9.7. Let $\phi: B \rightarrow A$ be a homomorphism of crystals, and assume $A$ is connected. Then every preimage $\phi^{-1}(\{a\})$ has the same cardinality, for all $a \in A$.

Proof. If $b=E_{i} a$ in $A$, the definitions imply that $E_{i}$ and $F_{i}$ are mutually inverse bijections between $\phi^{-1}(\{a\})$ and $\phi^{-1}(\{b\})$.

Corollary 9.8. Let $B$ be a crystal, and let $\alpha: B \rightarrow \mathbb{Q}(q, t)$ be a function that is constant on connected components of $B$. Let $\phi: B \rightarrow \mathbb{Z}_{+}^{n}$ be a crystal homomorphism. Assume that $B$ has finitely many elements of each weight, and set $\operatorname{ch} B=\sum_{b \in B} \alpha(b) \mathrm{wt}(b)$. Then $\operatorname{ch} B$ is a symmetric function, and the coefficient $c_{\lambda}(q, t)$ in the Schur function expansion $\operatorname{ch} B=\sum_{\lambda} c_{\lambda}(q, t) s_{\lambda}(x)$ is equal to

$$
\sum_{\substack{b \in B \\ \phi(b) \in \operatorname{Yam}(\lambda)}} \alpha(b) .
$$


Proof. We temporarily define $c_{\lambda}(q, t)$ by formula (73), and prove that $\operatorname{ch} B=$ $\sum_{\lambda} c_{\lambda}(q, t) s_{\lambda}(x)$, with $c_{\lambda}(q, t)$ so defined. Let $B^{(\lambda)}$ be the set of elements $b \in B$ such that $R(\phi(B))$ has shape $\lambda$. Let $V_{\lambda}$ be the crystal consisting of reading words $w(T)$ for $T \in \operatorname{SSYT}(\lambda)$; it is a connected component of the crystal $\mathbb{Z}_{+}^{n}$. Let $T_{0} \in \operatorname{SSYT}(\lambda)$ be the unique tableau such that $w_{0}=w\left(T_{0}\right)$ is Yamanouchi.

Now, $R \circ \phi$ is a crystal homomorphism from $B^{(\lambda)}$ onto $V_{\lambda}$, and the preimage of $w_{0}$ is $(R \circ \phi)^{-1}\left(\left\{w_{0}\right\}\right)=\{b \in B: \phi(b) \in \operatorname{Yam}(\lambda)\}$. We have defined $c_{\lambda}(q, t)$ to be the sum of $\alpha(b)$ over all $b$ in this set. Since $\alpha$ is constant on components of $B^{(\lambda)}$, Proposition 9.7 implies that for every $w \in V_{\lambda}$, the sum of $\alpha(b)$ over all $b \in(R \circ \phi)^{-1}(\{w\})$ is equal to $c_{\lambda}(q, t)$. Now, $s_{\lambda}(x)=\sum_{w \in V_{\lambda}} x^{w}=\sum_{w \in V_{\lambda}} \mathrm{wt}(w)$, and therefore $\operatorname{ch} B^{(\lambda)}=c_{\lambda}(q, t) s_{\lambda}(x)$. But $B$ is the disjoint union of its subsets $B^{(\lambda)}$, and summing over them all yields $\operatorname{ch} B=\sum_{\lambda} c_{\lambda}(q, t) s_{\lambda}(x)$.

Proof of Proposition 9.2. We will construct a crystal structure on the set $\Sigma_{\mu}$ of all fillings $\sigma: \mu \rightarrow \mathbb{Z}_{+}$, with the following properties. First, the weight function is the obvious one, $\operatorname{wt}(\sigma)=x^{\sigma}$. Second, the map $\phi: \Sigma_{\mu} \rightarrow \mathbb{Z}_{+}^{n}$ defined by $\phi(\sigma)=R(w(\sigma))$ is a crystal homomorphism, where $w(\sigma)$ denotes the reading word of $\sigma$. Third, $q^{\operatorname{inv}(\sigma)} t^{\operatorname{maj}(\sigma)}$ is constant on each component of $\Sigma_{\mu}$. Then Proposition 9.2 is a special case of Corollary 9.8 .

For simplicity, we define the crystal operators $E_{1}, F_{1}$. The definition of $E_{i}, F_{i}$ is the same with 1,2 replaced by $i, i+1$ in what follows. Fix the list $u_{1}, \ldots, u_{n}$ of all the cells of $\mu$ in reading order. Since $\mu$ has two columns, there is an index $k_{0}$ such that $u_{k}$ attacks $u_{k+1}$ for all $k_{0} \leq k<n$, and these are the only attacking pairs. Call $u_{k_{0}}, \ldots, u_{n}$ the attack zone.

If $E_{1} w(\sigma)=0$ (in $\mathbb{Z}_{+}^{n}$ ), we define $E_{1} \sigma=0$. Otherwise, let $u_{k}$ be the cell such that $\sigma\left(u_{k}\right)$ is the 2 in $w(\sigma)$ that would be changed to a 1 by applying the standard crystal operator $E_{1}$. If $k \leq n-2$ and $u_{k}, u_{k+1}, u_{k+2}$ are in the attack zone and $\sigma\left(u_{k}\right) \sigma\left(u_{k+1}\right) \sigma\left(u_{k+2}\right)=221$, then $w(\sigma)$ has the form $y 221 z$, and we define $E_{1} \sigma$ by $w\left(E_{1} \sigma\right)=y 211 z$. Otherwise, let $j \leq k$ be the smallest index such that $k-j$ is even, $u_{j}, u_{j+1}, \ldots, u_{k}$ is contained in the attack zone if $j \neq k$, and $\sigma\left(u_{j}\right) \sigma\left(u_{j+1}\right) \cdots \sigma\left(u_{k}\right)=2121 \cdots 2$. Then $w(\sigma)$ has the form $y 2121 \cdots 2 z$ and we define $E_{1} \sigma$ by $w\left(E_{1} \sigma\right)=y 1212 \cdots 1 z$.

If $F_{1} w(\sigma)=0$ (in $\mathbb{Z}_{+}^{n}$ ), we define $F_{1} \sigma=0$. Otherwise, let $u_{k}$ be the cell such that $\sigma\left(u_{k}\right)$ is the 1 in $w(\sigma)$ that would be changed to a 2 by applying the standard crystal operator $F_{1}$. If $k \geq 2$ and $u_{k-2}, u_{k-1}, u_{k}$ are in the attack zone and $\sigma\left(u_{k-2}\right) \sigma\left(u_{k-1}\right) \sigma\left(u_{k}\right)=211$, then $w(\sigma)$ has the form $y 211 z$, and we define $F_{1} \sigma$ by $w\left(F_{1} \sigma\right)=y 221 z$. Otherwise, let $l \geq k$ be the largest index such that $l-k$ is even, $u_{k}, u_{k+1}, \ldots, u_{l}$ is contained in the attack zone if $k \neq l$, and $\sigma\left(u_{k}\right) \sigma\left(u_{k+1}\right) \cdots \sigma\left(u_{l}\right)=1212 \cdots 1$. Then $w(\sigma)$ has the form $y 1212 \cdots 1 z$ and we define $F_{1} \sigma$ by $w\left(F_{1} \sigma\right)=y 2121 \cdots 2 z$.

Obviously, $E_{i}, F_{i}$ behave correctly with respect to weights. We must verify that $E_{i} \sigma=\tau$ if and only if $F_{i} \tau=\sigma$. It suffices to consider $i=1$. Suppose that $E_{1} \sigma=\tau$. We are in one of two cases. In the first case, the reading words are

$$
w(\sigma)=y 221 z \underset{E_{1}}{\longrightarrow} y 211 z=w(\tau) .
$$

The first unmatched 2 in $w(\sigma)$ is at the beginning of the indicated subsequence 221. It follows that the last unmatched 1 in $w(\tau)$ is at the end of the indicated subsequence 211. We are in the first case of the rule for $F_{1} \tau$, so $F_{1} \tau=\sigma$. In the 
second case of the rule for $E_{1} \sigma$, the reading words are

$$
w(\sigma)=y 2121 \cdots 2 z \underset{E_{1}}{\longrightarrow} y 1212 \cdots 1 z=w(\tau) .
$$

The first unmatched 2 in $w(\sigma)$ is at the end of the indicated subsequence $2121 \cdots 2$. Therefore the last unmatched 1 in $w(\tau)$ is at the beginning of the indicated subsequence $1212 \cdots 1$. Moreover, $y$ does not end with 21 in the attack zone, since the index $j$ was minimal. Also, it cannot happen that the subsequence $1212 \cdots 1$ is in the attack zone and $z$ begins with 21 , also in the attack zone, since that would put us in the first case of the rule for $E_{1} \sigma$. Hence we are in the second case of the rule for $F_{1} \tau$, and $F_{1} \tau=\sigma$.

The proof that $F_{1} \tau=\sigma$ implies $E_{1} \sigma=\tau$ is entirely similar and will be omitted.

Next we show that the operators $E_{i}, F_{i}$ preserve $\operatorname{inv}(\sigma)$ and $\operatorname{maj}(\sigma)$. In fact, we will show that they preserve $|\operatorname{Inv}(\sigma)|$ and $\operatorname{Des}(\sigma)$. The only inversions and descents that might be affected involve entries in $\{i-1, i, i+1, i+2\}$, so it suffices to consider $i=2$. By the crystal axioms, it suffices to consider the operator $E_{2}$. In the first case of the rule for $E_{2} \sigma$, the relevant subsequence 332 of $w(\sigma)$ occupies cells in $\mu$ forming one of the configurations

$$
\begin{array}{|l|l|}
\cline { 2 - 2 } & x \\
\hline 3 & 3 \\
\hline 2 & y \\
\hline
\end{array} \text { or } \begin{array}{|l|l|}
\hline x & 3 \\
\hline 3 & 2 \\
\hline y & \multicolumn{1}{|c}{} \\
\hline
\end{array}
$$

where $x$ or $y$ may be missing. If they are present, $x \neq 3$ and $y \neq 2$, because the first 3 in the subsequence 332 is the first unmatched 3 in $w$. Hence changing the middle 3 in 332 to a 2 does not change $\operatorname{Des}(\sigma)$, and it clearly does not change $|\operatorname{Inv}(\sigma)|$.

In the second case of the rule, we have a subsequence $3232 \cdots 3$ of the type

$$
\begin{array}{|l|l|}
\hline x & y \\
\hline 3 & 2 \\
\hline 3 & 2 \\
\hline 3 & z \\
\hline w &
\end{array} \quad \begin{array}{|l|l|}
n & x \\
\hline y & 3 \\
\hline 2 & 3 \\
\hline 2 & 3 \\
\hline z & w \\
\hline
\end{array} \text {. }
$$

For simplicity, we have illustrated the situation with a subsequence of length 5, although the actual picture might have more or fewer rows \begin{tabular}{|l|l|l|l|l}
3 & 2 & or & 2 & 3
\end{tabular} . Again, some of $x, y, z, w$ might be missing. If they are present, then $x, y \neq 3$ and $z, w \neq 2$. To see this, note that $y=3$ or $x=3, y \neq 2$ would contradict the fact that the bottom 3 is the first unmatched 3 in $w(\sigma)$, while $x=3, y=2$ would contradict the minimality of the index $j$. Similarly, $z=2$ or $w=2, z \neq 3$ contradicts unmatchedness, while $w=2, z=3$ would put us in the first case of the rule for $E_{2} \sigma$. Given that $x, y \neq 3$ and $z, w \neq 2$, it is easy to see that exchanging 2's with 3 's in the subsequence $3232 \cdots 3$ leaves $\operatorname{Des}(\sigma)$ and $|\operatorname{Inv}(\sigma)|$ unchanged.

The last thing we need to prove is that $\phi(\sigma)=R(w(\sigma))$ defines a crystal homomorphism. Since $R$ is a crystal homomorphism, this follows if we show that $R\left(w\left(E_{i} \sigma\right)\right)=R\left(E_{i} w(\sigma)\right)$ and $R\left(w\left(F_{i} \sigma\right)\right)=R\left(F_{i} w(\sigma)\right)$. In other words, we must show that after applying our crystal operators to $\sigma$, we get a reading word which is jeu-de-taquin equivalent to the one we would have gotten by applying the standard crystal operators to $w(\sigma)$. Consider the operator $E_{1}$. In the first case of the rule, our operator gives y $211 z$, while the standard $E_{1}$ would give $y 121 z$. But $211 \approx 121$ is a Knuth relation, so this case is fine. In the second case of the rule, our operator gives $y 1212 \cdots 121 z$, while the standard $E_{1}$ would give $y 2121 \cdots 211$. In this case, the result follows from the fact that $1212 \cdots 121$ and $2121 \cdots 211$ have the same 
RSK insertion tableau, namely, the tableau with all 1's in row 1 and all 2's in row 2 . The same argument applies to $E_{i}$ by taking $i, i+1$ in place of 1,2 . The argument for $F_{i}$ is entirely similar.

Remark. Since the crystal operators preserve $\operatorname{Des}(\sigma)$, the proof shows that the analog of Proposition 9.2 holds for each of the functions $F_{\mu, D}(x ; q)$ in (22)-(23).

\section{Appendix: A new Proof of LLT symmetry}

In this appendix we give a purely combinatorial proof of Theorem 3.3 .

Recall the notation from \$3 We need to extend it to "super" analogs of the LLT polynomial $G_{\nu}(x ; q)$. Let $\mathcal{A}$ be a super alphabet, as in (29), and define a super tableau $T$ on a skew shape $\nu$ to be a function $T: \nu \rightarrow \mathcal{A}$, weakly increasing on each row and column, with the property that if $i$ is positive, then $T^{-1}(\{i\})$ is a horizontal strip (i.e., has no two cells in the same column) and if $\bar{i}$ is negative, then $T^{-1}(\{\bar{i}\})$ is a vertical strip (no two cells in the same row). A super tableau with positive entries is just an ordinary semistandard tableau. Let $\operatorname{SSYT}_{ \pm}(\nu)$ denote the set of super tableaux, and for a tuple $\boldsymbol{\nu}=\left(\nu^{(1)}, \ldots, \nu^{(k)}\right)$, define $\operatorname{SSYT}_{ \pm}(\boldsymbol{\nu})=$ $\operatorname{SSYT}_{ \pm}\left(\nu^{(1)}\right) \times \cdots \times \operatorname{SSYT}_{ \pm}\left(\nu^{(k)}\right)$.

For $u \in \nu^{(j)}$, define

$$
\beta(u)=j / k-c(u) .
$$

The fractional part of $\beta$ determines $j$; hence $\beta(u)=\beta(v)$ if and only if $u$ and $v$ lie on a common diagonal $c(u)=c(v)$ in the same shape $\nu^{(j)}$. The content reading order is the unique total ordering on the cells of $\bigsqcup \boldsymbol{\nu}$ such that $\beta$ is weakly increasing, and cells with $\beta(u)=\beta(v)$ increase upward and to the right along diagonals. (Under the identification in the proof of Proposition 3.4 between fillings of $\mu$ and semistandard tableaux on tuples of ribbons, the content reading order corresponds to the reading order defined previously for fillings.) Given $u$ preceding $v$ in the content reading order, define entries $T(u)$ and $T(v)$ in a super tableau $T \in \operatorname{SSYT}_{ \pm}(\boldsymbol{\nu})$ to form an inversion if

$$
T(u)>T(v) \quad \text { or } \quad T(u)=T(v) \in \mathbb{Z}_{-}, \quad \text { and } \quad 0<\beta(v)-\beta(u)<1 .
$$

Let $\operatorname{inv}(T)$ be the number of inversions in $T$ and define

$$
\tilde{G}_{\boldsymbol{\nu}}(x, y ; q) \underset{\mathrm{def}}{=} \sum_{T \in \operatorname{SSYT}_{ \pm}(\boldsymbol{\nu})} q^{\operatorname{inv}(T)} z^{T}
$$

where $z_{i}=x_{i}$ for $i$ positive, $z_{\bar{i}}=y_{i}$ for $\bar{i}$ negative, as in (31). For semistandard tableaux with positive letters, (78) is equivalent to our original definition of inversions in $T$ and hence

$$
\tilde{G}_{\boldsymbol{\nu}}(x, 0 ; q)=G_{\boldsymbol{\nu}}(x ; q) .
$$

A semistandard tableau $S$ is standard if it is a bijection $S: \bigsqcup \boldsymbol{\nu} \rightarrow\{1, \ldots, n\}$, where $n=|\boldsymbol{\nu}|=\sum_{j}\left|\nu^{(j)}\right|$. Denote the set of standard tableau by $\operatorname{SYT}(\boldsymbol{\nu})$. Note that every $\nu^{(j)}$ is a horizontal strip if and only if the labelling of the cells of $\boldsymbol{\nu}$ from 1 to $n$ in increasing content reading order is a standard tableau. Similarly, every $\nu^{(j)}$ is a vertical strip if and only if the labelling of $\boldsymbol{\nu}$ in decreasing content reading order is standard. It follows that every super tableau $T \in \operatorname{SSYT}_{ \pm}(\boldsymbol{\nu})$ has a unique standardization $S \in \operatorname{SYT}(\boldsymbol{\nu})$ such that $T \circ S^{-1}$ is weakly increasing, and for $x \in \mathcal{A}$, the entries of $S$ on $T^{-1}(\{x\})$ are increasing in content reading order if $x$ is positive, 
decreasing if $x$ is negative. Using (78), we see that $T(u), T(v)$ form an inversion if and only if $S(u), S(v)$ do. Hence $\operatorname{inv}(T)=\operatorname{inv}(S)$.

Define the descent set $D(S) \subseteq\{1, \ldots, n-1\}$ of a standard tableau $S \in \operatorname{SYT}(\boldsymbol{\nu})$ by

$$
D(S)=\left\{i: S^{-1}(i+1) \text { precedes } S^{-1}(i) \text { in the content reading order }\right\} .
$$

If $S$ is the standardization of $T$, then $a=T \circ S^{-1}:\{1, \ldots, n\} \rightarrow \mathcal{A}$ is weakly increasing and satisfies the additional conditions that $a(i)=a(i+1) \in \mathbb{Z}_{+}$implies $i \notin D(S)$ and $a(i)=a(i+1) \in \mathbb{Z}_{-}$implies $i \in D(S)$. Conversely, if $a:\{1, \ldots, n\} \rightarrow \mathcal{A}$ satisfies these conditions, then $T=a \circ S$ is a super tableau, and its standardization is $S$. Comparing the definitions (31) and (79), we see that

$$
\tilde{G}_{\boldsymbol{\nu}}(x, y ; q)=\sum_{S \in \operatorname{SYT}(\boldsymbol{\nu})} q^{\operatorname{inv}(S)} \tilde{Q}_{n, D(S)}(x, y) .
$$

Setting $y=0$, we deduce as a special case that

$$
G_{\boldsymbol{\nu}}(x ; q)=\sum_{S \in \operatorname{SYT}(\boldsymbol{\nu})} q^{\operatorname{inv}(S)} Q_{n, D(S)}(x) .
$$

Lemma 10.1. Let $\boldsymbol{\nu}^{\prime}$ be obtained from $\nu$ by transposing each $\nu^{(j)}$ and reversing the tuple. Then $G_{\boldsymbol{\nu}^{\prime}}(x ; q)$ is a symmetric function if $G_{\boldsymbol{\nu}}(x ; q)$ is.

Proof. Assume $G_{\boldsymbol{\nu}}(x ; q)$ is symmetric. Then (81), (82) and Proposition 4.2 imply that $\tilde{G}_{\boldsymbol{\nu}}(x, y ; q)=\omega_{Y} G_{\boldsymbol{\nu}}[X+Y ; q]$ is symmetric in $x$ and $y$ separately. Hence $\tilde{G}_{\nu}(0, y ; q)$ is symmetric.

If $u$ is a cell in $\boldsymbol{\nu}$, denote by $u^{\prime}$ the cell in $\boldsymbol{\nu}^{\prime}$ corresponding to $u$ under the operation of transposing and reversing $\boldsymbol{\nu}$. We have $c\left(u^{\prime}\right)=-c(u)$, and if $u \in \nu^{(j)}$, then $u^{\prime} \in\left(\nu^{\prime}\right)^{k+1-j}$. Hence $\beta\left(u^{\prime}\right)=(k+1) / k-\beta(u)$. Now, $\tilde{G}_{\nu}(0, y ; q)$ is a generating function for super tableaux with negative entries. Given $T \in \operatorname{SSYT}\left(\boldsymbol{\nu}^{\prime}\right)$, define $\bar{T} \in \operatorname{SSYT}_{ \pm}(\boldsymbol{\nu})$ by $\bar{T}(u)=\overline{T\left(u^{\prime}\right)}$. Clearly $T \mapsto \bar{T}$ is a bijection from $\operatorname{SSYT}\left(\boldsymbol{\nu}^{\prime}\right)$ to the set of super tableaux of shape $\boldsymbol{\nu}$ with only negative entries. As we are free to do, we choose the ordering denoted $<_{1}$ in (30) of the super alphabet $\mathcal{A}$, so $\overline{1}<\overline{2}<\cdots$. Then we see that cells $u, v$ in $\boldsymbol{\nu}$ satisfy $0<\beta(v)-\beta(u)<1$ if and only if $v^{\prime}, u^{\prime}$ satisfy $0<\beta\left(u^{\prime}\right)-\beta\left(v^{\prime}\right)<1$, and for each such pair of cells, $T(u), T(v)$ form an inversion in $T$ if and only if the corresponding entries $\bar{T}\left(v^{\prime}\right)=\overline{T(v)}, \bar{T}\left(u^{\prime}\right)=\overline{T(u)}$ do not form an inversion in $\bar{T}$. Hence $\operatorname{inv}(\bar{T})=m-\operatorname{inv}(T)$, where $m$ is the number of pairs of cells $(u, v)$ in $\boldsymbol{\nu}$ satisfying $0<\beta(v)-\beta(u)<1$. It follows that

$$
G_{\nu^{\prime}}(y ; q)=q^{m} \tilde{G}_{\nu}\left(0, y ; q^{-1}\right),
$$

so $G_{\boldsymbol{\nu}^{\prime}}(x ; q)$ is symmetric.

Remark. The proof actually shows that $G_{\boldsymbol{\nu}^{\prime}}(x ; q)=q^{m} \omega G_{\nu}\left(x ; q^{-1}\right)$.

We now prove Theorem 3.3 by means of a series of reductions. It suffices to prove that the LLT polynomial $G_{\boldsymbol{\nu}}(x ; q)$ is symmetric in $x_{i}$ and $x_{i+1}$, for each $i$. Given a tableau $T \in \operatorname{SSYT}(\boldsymbol{\nu})$, let $\boldsymbol{\rho}=\left(\rho^{(1)}, \ldots, \rho^{(k)}\right)=T^{-1}(\{i, i+1\})$ and $S=\left.T\right|_{\boldsymbol{\rho}}$, so $S$ is the part of $T$ formed by entries $i$ and $i+1$. Let $U=\left.T\right|_{\nu \backslash \rho}$ be the rest of $T$. Note that $\boldsymbol{\rho}$ is a tuple of skew shapes, and for $\boldsymbol{\rho}$ and $U$ fixed, every semistandard tableau $S \in \operatorname{SSYT}(\boldsymbol{\rho})$ occurs for a unique $T$. Moreover,

$$
q^{\operatorname{inv}(T)} x^{T}=q^{i(\boldsymbol{\rho}, U)} x^{U} \cdot q^{\operatorname{inv}(S)} x^{S},
$$


where $i(\boldsymbol{\rho}, U)$ is a constant independent of $S$. This holds because for entries $x=$ $U(u) \notin\{i, i+1\}$ and $y=S(v) \in\{i, i+1\}$, the condition $x>y$ is independent of $y$. Partitioning the defining sum in (21) into smaller sums for each $(\boldsymbol{\rho}, U)$, we reduce the symmetry problem to the case of shapes $\boldsymbol{\rho}$ and tableaux $S \in \operatorname{SSYT}(\boldsymbol{\rho})$ with entries in a two-element set $\{i, i+1\}$.

We can now assume that each $\nu^{(j)}$ has at most two cells in each column, and we can evaluate $G_{\boldsymbol{\nu}}(x ; q)$ in just two variables $x=x_{1}, x_{2}$. Consider a column with two cells $\{u, v\}$ in $\nu^{(j)}$, say with $v$ above $u$; in every tableau $T \in \operatorname{SSYT}(\boldsymbol{\nu})$, we must have $T(u)=1, T(v)=2$.

$$
\begin{array}{|ll|l|}
\hline v & & 2 \\
\hline u & & \rightarrow \\
\hline
\end{array}
$$

Consider a third cell $w \in \nu^{(i)}$. Suppose that

$$
\text { either } c(w)=c(v)=c(u)+1 \text { and } i>j \text {, or } c(w)=c(u) \text { and } i<j \text {. }
$$

If $T(w)=1$, then $T(v)>T(w)$ is an inversion, but $T(w)=T(u)$ is not. Alternatively, if $T(w)=2$, then $T(w)>T(u)$ is an inversion, but $T(v)=T(w)$ is not. Hence the cells $u, v, w$ make a net contribution of 1 to inv $(T)$. One checks similarly that if $w$ does not satisfy (85), then the contribution to $\operatorname{inv}(T)$ from $u, v, w$ is zero, independent of $T(w)$. Let now $\boldsymbol{\rho}$ be the shape that remains upon deleting all two-cell columns from $\boldsymbol{\nu}$, and let $S=\left.T\right|_{\rho}$. Note that each $\rho^{(j)}$ is a skew shape, and we get every $S \in \operatorname{SSYT}(\boldsymbol{\rho})$ as the restriction of a unique $T$. The preceding observations show that

$$
q^{\operatorname{inv}(T)} x^{T}=q^{h(\boldsymbol{\nu})}\left(x_{1} x_{2}\right)^{m} \cdot q^{\operatorname{inv}(S)} x^{S},
$$

where $h(\boldsymbol{\nu})$ is a constant independent of $S$ and $m$ is the number of two-cell columns in $\boldsymbol{\nu}$. This reduces the problem to the case where each $\nu^{(j)}$ is a horizontal strip.

Applying Lemma 10.1, we reduce to the case where each $\nu^{(j)}$ is a vertical strip. Applying once more the same reductions that we used above for general $\boldsymbol{\nu}$, we reach the case that each $\nu^{(j)}$ is a disconnected union of single cells. Then the numbers $\beta(u)$ in (77) are distinct for all cells $u \in \bigsqcup \boldsymbol{\nu}$, and every function $T: \boldsymbol{\nu} \rightarrow\{1,2\}$ is a semistandard tableau. Thus we come down to the following lemma.

Lemma 10.2. Let $\beta_{1}<\beta_{2}<\cdots<\beta_{n}$ be arbitrary real numbers. For every word $w=w_{1} w_{2} \ldots w_{n}$ with $w_{i} \in\{1,2\}$, define

$$
\operatorname{inv}_{\beta}(w)=\mid\left\{(i<j): w_{j}>w_{i} \text { and } \beta_{j}-\beta_{i}<1\right\} \mid .
$$

Then the polynomial

$$
G_{\beta}\left(x_{1}, x_{2} ; q\right) \underset{\overline{d e f}}{=} \sum_{w \in\{1,2\}^{n}} q^{\operatorname{inv}_{\beta}(w)} \prod_{i=1}^{n} x_{w_{i}}
$$

is symmetric in $x_{1}$ and $x_{2}$.

Proof. Let $r=\left|\left\{i<n: \beta_{n}-\beta_{i}<1\right\}\right|$. We will prove the lemma by double induction on $n$ and $r$. The case $n=0$ is trivial, since $G_{\emptyset}(x ; q)=1$. If $r=0$, then $w_{n}$ forms no inversions with the rest of the word, and we have

$$
G_{\beta}(x ; q)=\left(x_{1}+x_{2}\right) G_{\left(\beta_{1}, \ldots, \beta_{n-1}\right)}(x ; q),
$$

which is symmetric by induction on $n$.

If $r>0$, define $\alpha_{i}=\beta_{i}$ for $i<n$, and fix $\alpha_{n}$ such that $\beta_{n-r}+1<\alpha_{n}<\beta_{n-r+1}+$ 1. By the definition of $r$, we have $\beta_{n}<\beta_{n-r}+1$, hence $\alpha_{n}>\beta_{n}>\beta_{n-1}=\alpha_{n-1}$, 
so $\alpha$ is an increasing sequence. By construction, $\left|\left\{i<n: \alpha_{n}-\alpha_{i}<1\right\}\right|=r-1$, so $G_{\alpha}(x ; q)$ is symmetric by induction on $r$.

We now compare $\operatorname{inv}_{\alpha}(w)$ and $\operatorname{inv}_{\beta}(w)$ for an arbitrary word $w$. In positions $i<j<n, w_{i}$ and $w_{j}$ form an inversion with respect to $\alpha$ if and only if they form an inversion with respect to $\beta$. This also holds for $j=n$ and $i \neq n-r$, since $\beta_{n}-\beta_{i}<1$ if and only if $i \geq n-r$, and $\alpha_{n}-\alpha_{i}<1$ if and only if $i \geq n-r+1$. Hence

$$
\operatorname{inv}_{\beta}(w)=\operatorname{inv}_{\alpha}(w)+ \begin{cases}1 & \text { if } w_{n-r}=2, w_{n}=1, \\ 0 & \text { otherwise. }\end{cases}
$$

Now, if $w_{n-r}=2$ and $w_{n}=1$, then $w_{n-r}$ and $w_{n}$ together form exactly one inversion with each $w_{i}$ for $n-r<i<n$ and no inversions with $w_{i}$ for $i<n-r$. This holds for inversions with respect to either $\alpha$ or $\beta$. Hence the contribution to $G_{\beta}(x ; q)$ from terms indexed by such words $w$ is $q^{r} x_{1} x_{2} G_{\gamma}(x ; q)$, where $\gamma=\left(\beta_{1}, \ldots, \beta_{n-r-1}, \beta_{n-r+1}, \ldots, \beta_{n-1}\right)$, while the contribution to $G_{\alpha}(x ; q)$ from the same words $w$ is $q^{r-1} x_{1} x_{2} G_{\gamma}(x ; q)$. The contributions to $G_{\alpha}(x ; q)$ and $G_{\beta}(x ; q)$ from all other words are equal. Hence

$$
G_{\beta}(x ; q)-G_{\alpha}(x ; q)=\left(q^{r}-q^{r-1}\right) x_{1} x_{2} G_{\gamma}(x ; q) .
$$

Since $G_{\gamma}(x ; q)$ is symmetric by induction on $n$ and $G_{\alpha}(x ; q)$ is symmetric by induction on $r$, the lemma is proved, and the proof of Theorem 3.3 is complete.

\section{REFERENCES}

[1] Lynne M. Butler, Combinatorial properties of partially ordered sets associated with partitions and finite abelian groups, Ph.D. thesis, Massachusetts Institute of Technology, 1986.

[2] _ Subgroup lattices and symmetric functions, Mem. Amer. Math. Soc. 112 (1994), no. 539, vi+160. MR.1223236 (95e:05122)

[3] Christophe Carré and Bernard Leclerc, Splitting the square of a Schur function into its symmetric and antisymmetric parts, J. Algebraic Combin. 4 (1995), no. 3, 201-231. MR1331743 (97b:05165)

[4] Susanna Fishel, Statistics for special q,t-Kostka polynomials, Proc. Amer. Math. Soc. 123 (1995), no. 10, 2961-2969. MR1264811 (95m:05244)

[5] A. M. Garsia and J. Remmel, Plethystic formulas and positivity for q, t-Kostka coeffcients, Mathematical essays in honor of Gian-Carlo Rota (Cambridge, MA, 1996), Birkhäuser Boston, Boston, MA, 1998, pp. 245-262. MR1627327 (99j:05189d)

[6] A. M. Garsia and G. Tesler, Plethystic formulas for Macdonald q, t-Kostka coefficients, Adv. Math. 123 (1996), no. 2, 144-222. MR1420484 (99j:05189e)

[7] A. M. Garsia and M. Zabrocki, Polynomiality of the q,t-Kostka revisited, Algebraic combinatorics and computer science, Springer Italia, Milan, 2001, pp. 473-491, arXiv:math.QA/0008199. MR1854489 (2002h:05160)

[8] J. Haglund, A combinatorial model for the Macdonald polynomials, Proc. Nat. Acad. Sci. U.S.A. 101 (2004), no. 46, 16127-16131. MR2114585

[9] J. Haglund, M. Haiman, N. Loehr, J. B. Remmel, and A. Ulyanov, A combinatorial formula for the character of the diagonal coinvariants, Duke Math. J. 126 (2005), no. 2, 195-232, arXiv:math.CO/0310424. MF 2115257

[10] Mark Haiman, Macdonald polynomials and geometry, New perspectives in geometric combinatorics (Billera, Björner, Greene, Simion, and Stanley, eds.), MSRI Publications, vol. 38, Cambridge University Press, 1999, pp. 207-254. MR.1731818 (2001k:05203)

[11] _ Hilbert schemes, polygraphs and the Macdonald positivity conjecture, J. Amer. Math. Soc. 14 (2001), no. 4, 941-1006, arXiv:math.AG/0010246. MR.1839919 (2002c:14008)

[12] _ Notes on Macdonald polynomials and the geometry of Hilbert schemes, Symmetric functions 2001: surveys of developments and perspectives, NATO Sci. Ser. II Math. Phys. Chem., vol. 74, Kluwer Acad. Publ., Dordrecht, 2002, pp. 1-64. MR.2059359 
[13] M. Kashiwara, On crystal bases of the Q-analogue of universal enveloping algebras, Duke Math. J. 63 (1991), no. 2, 465-516. MR.1115118 (93b:17045)

[14] M. Kashiwara, T. Miwa, and E. Stern, Decomposition of q-deformed Fock spaces, Selecta Math. (N.S.) 1 (1995), no. 4, 787-805, arXiv:q-alg/9508006. MR.1383585 (97c:17021)

[15] Anatol N. Kirillov and Masatoshi Noumi, Affine Hecke algebras and raising operators for Macdonald polynomials, Duke Math. J. 93 (1998), no. 1, 1-39, arXiv:q-alg/9605004. MR.1620075 (99j:05189a)

[16] Friedrich Knop, Integrality of two variable Kostka functions, J. Reine Angew. Math. 482 (1997), 177-189, arXiv:q-alg/9603027. MR1427661 (99j:05189c)

[17] Friedrich Knop and Siddhartha Sahi, A recursion and a combinatorial formula for Jack polynomials, Invent. Math. 128 (1997), no. 1, 9-22, arXiv:q-alg/9610016. MR 1437493 (98k:33040)

[18] L. Lapointe and J. Morse, Tableaux statistics for two part Macdonald polynomials, Algebraic combinatorics and quantum groups, World Sci. Publishing, River Edge, NJ, 2003, pp. 61-84, arXiv:math.CO/9812001. MF/2035130

[19] Luc Lapointe and Luc Vinet, Rodrigues formulas for the Macdonald polynomials, Adv. Math. 130 (1997), no. 2, 261-279, arXiv:q-alg/9607025. MR1472319 (99e:05128)

[20] Alain Lascoux, Bernard Leclerc, and Jean-Yves Thibon, Ribbon tableaux, Hall-Littlewood functions, quantum affine algebras, and unipotent varieties, J. Math. Phys. 38 (1997), no. 2, 1041-1068, arXiv:q-alg/9512031. MF1434225 (98c:05167)

[21] Alain Lascoux and Marcel-Paul Schützenberger, Sur une conjecture de H. O. Foulkes, C. R. Acad. Sci. Paris Sér. A-B 286 (1978), no. 7, A323-A324. MR0472993 (57:12672)

[22] _ Croissance des polynômes de Foulkes-Green, C. R. Acad. Sci. Paris Sér. A-B 288 (1979), no. 2, A95-A98. MR.0524758 (80d:20052)

[23] L Le monoïde plaxique, Noncommutative structures in algebra and geometric combinatorics (Naples, 1978), CNR, Rome, 1981, pp. 129-156. MR0646486 (83g:20016)

[24] Bernard Leclerc and Jean-Yves Thibon, Littlewood-Richardson coefficients and KazhdanLusztig polynomials, Combinatorial methods in representation theory (Kyoto, 1998), Adv. Stud. Pure Math., vol. 28, Kinokuniya, Tokyo, 2000, pp. 155-220, arXiv:math.QA/9809122. MR $1864481(2002 \mathrm{k}: 20014)$

[25] I. G. Macdonald, A new class of symmetric functions, Actes du 20e Séminaire Lotharingien, vol. 372/S-20, Publications I.R.M.A., Strasbourg, 1988, pp. 131-171.

[26] _ Symmetric functions and Hall polynomials, second ed., The Clarendon Press, Oxford University Press, New York, 1995, with contributions by A. Zelevinsky, Oxford Science Publications. MR.1354144(96h:05207)

[27] Laurent Manivel, Symmetric functions, Schubert polynomials and degeneracy loci, SMF/AMS Texts and Monographs, vol. 6, American Mathematical Society, Providence, RI, 2001, Translated from the 1998 French original by John R. Swallow, Cours Spécialisés [Specialized Courses], 3. MR1852463 (2002h:05161)

[28] Siddhartha Sahi, Interpolation, integrality, and a generalization of Macdonald's polynomials, Internat. Math. Res. Notices 10 (1996), 457-471. MR1399411 (99j:05189b)

[29] M.-P. Schützenberger, La correspondance de Robinson, Combinatoire et représentation du groupe symétrique (Actes Table Ronde CNRS, Univ. Louis-Pasteur Strasbourg, Strasbourg, 1976), Springer, Berlin, 1977, Lecture Notes in Mathematics, Vol. 579, pp. 59-113. MR0498826 (58:16863)

[30] Richard P. Stanley, Enumerative combinatorics. Vol. 2, Cambridge University Press, Cambridge, 1999, With a foreword by Gian-Carlo Rota and appendix 1 by Sergey Fomin. MR.1676282 (2000k:05026)

[31] Marc A. A. van Leeuwen, Some bijective correspondences involving domino tableaux, Electron. J. Combin. 7 (2000), Research Paper 35, 25 pp. (electronic), arXiv:math.CO/9909119. MR 1769066 (2001d:05195)

[32] Mike Zabrocki, Positivity for special cases of $(q, t)$-Kostka coefficients and standard tableaux statistics, Electron. J. Combin. 6 (1999), Research Paper 41, 36 pp. (electronic), arXiv:math.CO/9901016. MF1725705 (2001g:05101) 
Department of Mathematics, University of Pennsylvania, Philadelphia, Pennsylvania 19104-6395

E-mail address: jhaglund@math.upenn.edu

Department of Mathematics, University of California, Berkeley, California 974203840

E-mail address: mhaiman@math.berkeley.edu

Department of Mathematics, University of Pennsylvania, Philadelphia, Pennsylvania 19104-6395

E-mail address: nloehr@math.upenn.edu

Current address: Department of Mathematics, College of William \& Mary, Williamsburg, Virginia 23187-8795

E-mail address: nick@math.wm.edu 